\title{
Model-based, Mutation-driven Test-case Generation Via Heuristic-guided Branching Search
}

\author{
ANDREAS FELLNER, AIT Austrian Institute of Technology, TU Wien \\ WILLIBALD KRENN, RUPERT SCHLICK, and THORSTEN TARRACH, \\ AIT Austrian Institute of Technology \\ GEORG WEISSENBACHER, TU Wien
}

\begin{abstract}
This work introduces a heuristic-guided branching search algorithm for model-based, mutation-driven testcase generation. The algorithm is designed towards the efficient and computationally tractable exploration of discrete, non-deterministic models with huge state spaces. Asynchronous parallel processing is a key feature of the algorithm. The algorithm is inspired by the successful path planning algorithm Rapidly exploring Random Trees (RRT). We adapt RRT in several aspects towards test-case generation. Most notably, we introduce parametrized heuristics for start and successor state selection, as well as a mechanism to construct test cases from the data produced during the search.

We implemented our algorithm in the existing test-case generation framework MoMuT. We present an extensive evaluation of the proposed heuristics and parameters of the algorithm, based on a diverse set of demanding models obtained in an industrial context. In total, we continuously utilized $128 \mathrm{CPU}$ cores on three servers for several weeks to gather the experimental data presented. We show that branching search works well and the use of multiple heuristics is justified. With our new algorithm, we are now able to process models consisting of over 2,300 concurrent objects. To our knowledge, there is no other mutation-driven test-case generation tool that is able to process models of this magnitude.
\end{abstract}

CCS Concepts: $\bullet$ Software and its engineering $\rightarrow$ Empirical software validation; $\bullet$ Hardware $\rightarrow$ Defectbased test;

Additional Key Words and Phrases: Test case generation, model-based testing, mutation testing, search-based testing, heuristics, parallel search

\footnotetext{
This work has been conducted within the ENABLE-S3 project that has received funding from the ECSEL joint undertaking under grant agreement no 692455. This joint undertaking receives support from the European Union's Horizon 2020 Research and Innovation Programme and Austria, Denmark, Germany, Finland, Czech Republic, Italy, Spain, Portugal, Poland, Ireland, Belgium, France, Netherlands, United Kingdom, Slovakia, and Norway. ENABLE-S3 is funded by the Austrian Federal Ministry of Transport, Innovation and Technology (BMVIT) under the program "ICT of the Future" via FFG project number 853308 between May 2016 and April 2019. Furthermore, this work was partially supported by the Austrian National Research Network S11403-N23 (RiSE), the LogiCS doctoral program W1255-N23 of the Austrian Science Fund (FWF), by the Vienna Science and Technology Fund (WWTF) through the projects Heisenbugs project VRG11-005, by FFG projects number 845582 (TRUCONF) and 1821983 (AutoDrive).

Authors' addresses: A. Fellner, W. Krenn, R. Schlick, and T. Tarrach, AIT Austrian Institute of Technology, Giefinggasse 4, 1210 Vienna Austria; emails: \{andreas.fellner, willibald.krenn, rupert.schlick, thorsten.tarrach\}@ait.ac.at; G. Weissenbacher, Institute of Logic and Computation, Formal Methods in Systems Engineering, Favoritenstraße 9-11/192-4, A-1040 Vienna, Austria; email: georg.weissenbacher@tuwien.ac.at.

Permission to make digital or hard copies of part or all of this work for personal or classroom use is granted without fee provided that copies are not made or distributed for profit or commercial advantage and that copies bear this notice and the full citation on the first page. Copyrights for third-party components of this work must be honored. For all other uses, contact the owner/author(s).

(C) 2019 Copyright held by the owner/author(s).

1539-9087/2019/01-ART4

https://doi.org/10.1145/3289256
} 
ACM Reference format:

Andreas Fellner, Willibald Krenn, Rupert Schlick, Thorsten Tarrach, and Georg Weissenbacher. 2019. Modelbased, Mutation-driven Test-case Generation Via Heuristic-guided Branching Search. ACM Trans. Embed. Comput. Syst. 18, 1, Article 4 (January 2019), 28 pages.

https://doi.org/10.1145/3289256

\section{INTRODUCTION}

The aim of model-based testing (MBT) is to automatically create test cases for some system under test (SUT), based on a formal model of the system. The model usually reflects the specification of the SUT. Thus, MBT is a way of verifying whether the implemented system adheres to its specification.

Model-based mutation testing is a fault-based variant of MBT, where the generated test cases are guaranteed to detect implementations adhering to certain faulty versions of the specification. The idea here is to show that in implementing the system, the requirements were correctly understood and that the SUT is free of the faults that were injected into the specification. Faulty specifications are called mutants, hence the term model-based mutation testing (MBMT). MBMT is a semantically very rich test-case generation technique, but it is often considered infeasible due to the high overhead related to computing test sequences that detect discrepancies between the original specification and mutations of it. We present a test-case generation technique for MBMT that scales to large models from industry.

Our test-case generation tool MoMuT (www.momut.org) started out as a research prototype [2] and has since matured into a leading MBMT tool by applying it to multiple industrial case studies. The performance has improved tremendously over our initial prototypes. Starting from small toy models, we are now able to process industry-size models with more than 2,300 concurrently running state machines. This was made possible by a new exploration algorithm that is able to leverage the power of today's highly concurrent CPUs.

Killing Mutants. The quality of an MBMT test-case generator is measured by the number of mutants it kills. A mutant is killed if there is a path from the initial state of the model to a state that is affected by the mutation, in the sense that the mutation changes the behavior of the model at that state. To expose a fault and thereby a mutant, a test needs to achieve three things: It needs to execute the faulty part of the model (reachability), the mutant's state needs to deviate from the original model's state (infection), and the deviation needs to become visible to the outside (propagation).

The mutation-based testing community differentiates between two forms of killing: weak and strong $[13,27]$. Strong killing seeks a change in the observable behavior, whereas weak killing seeks a change in state (not requiring propagation). Weak killing is sometimes used in evaluating test suites, as it is a viable method to measure coverage and easier to evaluate than strong killing. As a test goal, although harder to achieve, strong killing must be pursued, because without propagation, the fault is not observed.

Test conformance. The tests we generate shall verify that all behaviors of the implementation are allowed by the specification. The model might under-specify the implementation, accepting many different implementations as correct. Therefore, we cannot require full equivalence of outputs to decide if a change in observable behavior actually violates the specification. Formal conformance theory can help with this. It is based on the question of what it means for an implementation to conform to a specification. Formal conformance testing, as presented in Reference [49], investigates theoretical methods to prove or disprove conformance. 
We chose input-output conformance (IOCO) as the basis of our MBMT approach, because it fits our event-based view on the interface of the system under test and supports under-specification. The semantics of IOCO is thoroughly defined in Reference [50] on labeled transition graphs. We instantiate these abstract semantics by showing how it can be connected to mutation testing of models, written in a widely used modeling formalism, such as UML. This enables specification engineers to both express models in an intuitive way and have well-defined conformance semantics.

Exploration. Our initial implementation used a straightforward algorithm that explored the state space randomly beginning from the initial state. When parallelized, this approach ignores any opportunity of coordination between the parallel random explorations. For our new implementation, we adopted a version of Rapidly exploring Random Trees (RRT), an algorithm that was very successful in task planning [36]. RRT works well in our setting. It basically works by exploring short paths into the state space and then starting anew, but not necessarily from the initial state; it can restart from any previously discovered state. This allows massive parallelization, because a large number of these short paths can be explored in parallel.

In this work, we compare several heuristics for choosing the starting states of new exploration paths and for guiding the exploration along certain paths. In particular, we make the following contributions:

- We lift and adapt the rapidly exploring the RRT algorithm from path planning, where it is highly successful and widely used, to the discrete verification world.

- We show that through branching search, we increase search performance and test-case quality.

- We propose and evaluate new search heuristics for discrete state spaces, including techniques based on distances and rarity of states.

- Using a range of case studies obtained in an industrial context, we show that MBMT scales to large models. To our knowledge no other MBMT tool can deal with models of this magnitude.

- We perform a thorough evaluation of our algorithmic framework and its parameters. We show that branching search is superior to performing no branching. We use statistical analysis to compare heuristics and show that multiple heuristics perform well.

- We show that several heuristics perform well on different models, although no single best one can be made out. Furthermore, we show that combining multiple heuristics independently discovers more mutants than each single heuristic on its own.

This article is based on Reference [20], introducing the following additions:

- We present how strong mutation killing on models can be related to conformance theory.

- We instantiate our algorithmic framework to perform classical breadth-first search in parallel.

- We present a new heuristic: CGoal.

- We extend our evaluation with additional models. The additional models are originally written in modeling languages not considered in Reference [20].

- We rerun the experiments with an extended set of models with additional mutant operators applied to all models.

- We add experiments measuring additional aspects of our algorithmic framework.

- We show how the results of the search can be transformed into test suites via a greedy and a shortest path method and compare them experimentally.

The article is organized as follows. In Section 2, we provide related research. In Section 3, we introduce the syntax and semantics of our modeling language. In Section 4, we present model-based 
mutation testing of reactive systems and show how our setting differs from classical mutation testing settings. In Section 5, we present the state space exploration algorithm, its parameters and heuristics that can be used to instantiate it, and how we create test cases. In Section 6, we present the industrial case study models used for our experiments. In Section 7, we present our experiments and results. In Section 8, we discuss threats to validity. In Section 9, we provide our conclusions and give an outlook to future work.

\section{RELATED RESEARCH}

Search-based testing techniques. We use a search-based technique to construct test cases. However, in contrast to classical search-based testing, we do not search over the input but the state space. A comprehensive overview of search-based testing is provided in Reference [40], both for the white-box and for black-box setting. A popular approach within search-based testing is casting test-case generation as an optimization problem [24, 32, 48, 52, 54]. Another popular technique within search-based testing is genetic programming for gradually improving test suite quality, starting from a random test suite $[22,38,53]$. Both techniques require executing/evaluating test cases multiple times, which is prohibitively expensive on our models.

Guided random testing. We use guided random testing to explore the state space of our models. Guided random testing is performed in Reference [37] by introducing multiple techniques to enhance random testing with static and dynamic analysis information. A classic automated white-box test-case generation tool is DART [25], which combines random testing with symbolic execution-based guidance. Furthermore, Randoop [41, 42] uses feedback from test executions to guide random automatic generation of Java unit tests. Whereas the above ideas are applied to code, we perform test-case generation on models. REDIRECT [45] is a model-based testing approach that applies guided random techniques to Simulink/Stateflow models. Apart from using a different class of models, the authors of Reference [45] target state and transition coverage, in contrast to the mutation coverage considered in this work.

Adaptive random testing $[14,16]$ aims to distribute test inputs uniformly across the input data space. This is a similar idea to RRT, which tries to uniformly cover state spaces. However, the inherent difference to our work is that in contrast to simple input values, inputs of reactive models are sequences of actions. Therefore, we work with an infinite input space that is not easily classified via some simple metric.

Rapidly exploring random trees. Rapidly exploring random trees [36] is a well established planning algorithm. RRT is mostly used in continuous domains $[6,21,28]$, since it heavily depends on distance metrics, which naturally arise in such domains. However, RRT was used in testing of hybrid systems [19] and to solve well defined discrete planning tasks in Reference [11]. We propose an RRT inspired search algorithm for a general-purpose discrete setting and a novel set of heuristics, some of which are not distance based.

Mutation testing. There are many different test coverage metrics, such as path, branch, or defuse coverage. Unlike control or dataflow-oriented measures, mutation coverage is a fault-based measure. Like search-based testing, mutation-based testing is an active area of research [17, 23, 43, 46, 47].

Mutation testing was introduced in the late 1970s by Demilio and Budd et al. [13] as a technique for measuring test-case adequacy. Since then, it is an active area of research [17, 23, 43, 46, 47]. A detailed survey of methods, problems, and developments over the field can be found in Reference [30]. The strength of mutation coverage was evaluated with empirical studies [7, 31].

Model-based testing. Model-based testing is a rich and diverse field of study. In Reference [51], a taxonomy of model-based testing methods is presented, and Reference [12] provides a detailed look 
at model-based testing of reactive systems. Many types of models have been targets of test-case generation, such as finite-state machines [15], Simulink [45], labeled transition systems [29], and SysML [26]. We perform test-case generation on Action System models. Due to its expressivity, the language can serve as both original and intermediary models. For example, UML state machine models can automatically be translated to this language by MoMuT. In Reference [5] a testing approach via symbolic refinement on action system models is presented. However, only very small models are processed in contrast to the models presented and processed in this work.

MoMuT. Our implementation is based on the MoMuT tool [2], which evolved from a long series of research $[3,4,33]$. We do not know of any tool but MoMuT that is able to process action systems of the size of our benchmark examples.

\section{THE MODELING LANGUAGE}

Our MBT approach is based on formal models given as action systems. The action system modeling formalism was initially proposed by Back [9] and is based on Dijkstra's guarded command language [18]. Action systems provide a good compromise between expressiveness and simplicity. Furthermore, they are general enough such that we can automatically map other modeling formalisms onto them, as it has been done with UML [33]. We provide a brief overview of action systems here, a more in-depth description of the formalism can be found in Reference [33].

\subsection{Syntax}

An action system is of the form

$$
\mid[\mathcal{V}: \mathcal{T} \leftarrow \mathcal{I} \text {; do } \mathcal{A} \text { od }] \mid,
$$

where $\mathcal{A}$ is an action and $\mathcal{V}$ is a finite set of variables with types in $\mathcal{T}$, initialized with values $\mathcal{I}$. Types are boolean, enumeration $\left(\left\{e_{1}, \ldots, e_{n}\right\}\right)$, integer $(n, m)$, or $\operatorname{list}(T, m)$. An enumeration type is a finite set of unique values $e_{1}, \ldots, e_{n}$. An integer type represents the interval $[n, m]$, and a list type represents all lists of maximum length $m$, the elements of which are of type $T$ (lists can contain lists). boolean is a specific enumeration type with the values $\{T, \perp\}$ and their usual semantics. All types are finite. Expressions can be formed using constants and variables, using the following operators: Boolean operators $(\wedge, \vee, \Rightarrow, \Leftrightarrow, \neg)$, comparison $(=,<,>, \leq, \geq)$, arithmetic operators $(+,-, *, /, \bmod , a b s)$, and list operators (head,tail,fold, $\in$,concatenation,length, $n i l)$.

An action is the composition of two other actions, a guarded command, an assignment, skip, or abort. Composed actions are formed via sequential, non-deterministic, or prioritized composition. Each guarded command and each assignment is labeled with a label $\ell$. Every labeled action is observable, controllable, or internal. We call observable and controllable labels visible and sometimes refer to observable/controllable action labels simply as observables and controllables. We use the convention that observable labels start with the prefix obs, controllable labels start with the prefix ctr, and all other labels are internal. The differentiation between these kinds of labels is an additional modeling tool, indicating whether an action models an external stimulus of a system, i.e., is controllable, models an event that can be observed from outside the system, i.e., is observable, or models a transition occurring inside the system that cannot be observed, i.e., is internal. These annotations have no effect on the semantics of action systems, but are important for killing mutants and interpreting sequences of action labels as tests.

Example 3.1. In Figure 1, we present a small running example. It models a simple coffee machine with a single controllable: ctr_coffee, representing a request of some external agent to the system to produce a cup of coffee. To produce a coffee, the model requires the machine to heat up, via the observable obs_heating, and then to pour the coffee, via the the observable obs_heating. The model is non-deterministic, allowing for heating to occur either once or twice before pouring. Thus, the 


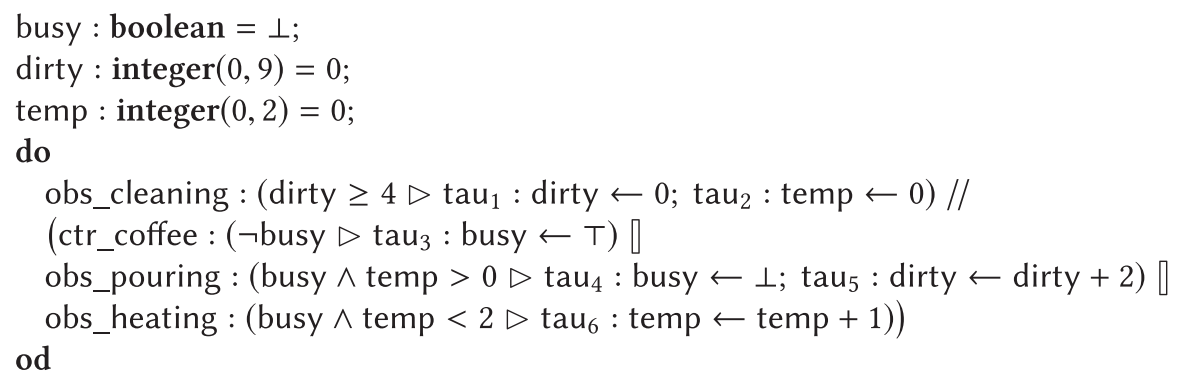

Fig. 1. Running example.

Table 1. Successor State Semantics

\begin{tabular}{lll}
\hline Action $(\mathrm{A})$ & Notation & $\mathbf{s u}(\mathrm{A}, \mathbf{l}, \mathbf{s})$ \\
\hline skip & skip & $\{(l, s)\}$ \\
abort & abort & $\emptyset$ \\
Assignment & $\ell: v \leftarrow e$ & $\{(l \cdot\langle\ell\rangle, s[v \mapsto e])\}$ \\
Guarded command & $\ell: g \triangleright A_{1}$ & $(s \mid=g) ? s u\left(A_{1}, l \cdot\langle\ell\rangle, s\right): \emptyset$ \\
Sequential Composition & $A_{1} ; A_{2}$ & $\bigcup\left(l^{\prime}, s^{\prime}\right) \in s u\left(A_{1}, l, s\right)\left(s u\left(A_{2}, l^{\prime}, s^{\prime}\right)\right)$ \\
Non-det Composition & $A_{1} \rrbracket A_{2}$ & $s u\left(A_{1}, l, s\right) \cup s u\left(A_{2}, l, s\right)$ \\
Prioritized Composition & $A_{1} / / A_{2}$ & $s u\left(A_{1}, l, s\right) \neq \emptyset ? s u\left(A_{1}, l, s\right): s u\left(A_{2}, l, s\right)$ \\
\hline
\end{tabular}

model represents a partial specification, not specifying exactly how much the machine needs to heat. Once the internal variable dirty is above a threshold, the observable action obs_cleaning is enabled. This action is prioritized over all other actions; therefore, a new coffee cannot be requested if dirty $\geq 4$ and obs_cleaning was not yet performed. Finally, we enumerate internal labels with the notation $\operatorname{tau}_{x}$.

Objects. We study models that are written in an object-oriented extension of action systems. However, all objects need to be created statically during initialization. Therefore, for the purpose of this work, we can view objects simply as a partition of variables. For an object, the object value is a Cartesian product of the values of its variables, together with a unique identifier of its deriving class. A more detailed discussion of object orientation in action systems can be found in Reference [10].

\subsection{Semantics}

States. A mapping of variables to values of their respective types is called a state s. Since all variables must have an initial value, every action system has a unique initial state $s_{l}$. Note that our state space is necessarily finite, because all integer variables have bounds and all lists a maximal length.

Small-step semantics. Given a predicate $P$ and state $s$, we say that $s$ satisfies $P$ and write $s \models P$ if $P$ evaluates to true under assignment $s$. The semantics of an action $A$ is defined by the successor function $s u(A, l, s)$ (see Table 1). su accepts an action $A$, a sequence of labels $l$ representing a path that is initially empty, and a state $s$. It returns a set of pairs, each pair $\left(l^{\prime}, s^{\prime}\right)$ consists of a sequence of labels $l^{\prime}$ and a state $s^{\prime}$, such that $l$ is a prefix of $l^{\prime}$. The labels describe the guards and assignments that lead from $s$ to $s^{\prime}$. We write $\operatorname{succ}_{\mathcal{A}}(s)$ for the set of successor states of $s\left(\left\{s^{\prime} \mid \exists l^{\prime} .\left(l^{\prime}, s^{\prime}\right) \in\right.\right.$ $\operatorname{su}(\mathcal{A}$, nil,$s)\})$. We write $\operatorname{succ}_{\mathcal{A}}(s, l)$ for the set of successor states of $s$ following the sequence of labels $l\left(\left\{s^{\prime} \mid\left(l, s^{\prime}\right) \in s u(\mathcal{A}\right.\right.$, nil, $\left.\left.s)\right\}\right)$. We use $\operatorname{path}_{\mathcal{A}}(s)$ as an abbreviation for $s u(\mathcal{A}$, nil, $s)$. For a tuple $\pi \in \operatorname{path}_{\mathcal{A}}(s)$, we use the notation $\pi . l$ to refer to its path and $\pi . s$ to refer to its state component. 
We call a state $s^{\prime}$ reachable if it is $s_{\iota}$ or $s^{\prime} \in \operatorname{succ}_{\mathcal{A}}(s)$ for some reachable state $s$. We call a labeled action with label $\ell$ reachable if there exists a reachable state $s$ such that $\ell$ is contained in the sequence $\pi . l$ for some $\pi \in \operatorname{path}_{\mathcal{A}}(s)$.

Example 3.2. In our running example from Figure 1 the initial state $s_{\iota}$ would be [busy $\mapsto$ $\perp$, dirty $\mapsto 0$, temp $\mapsto 0]$. We will represent the state as $[\perp, 0,0]$ for brevity and use this notation throughout the article. path $h_{\mathcal{A}}\left(s_{l}\right)=\left\{\left(\left\langle\operatorname{ctr} \_c o f f e e, \operatorname{tau}_{3}\right\rangle,[\mathrm{T}, 0,0]\right)\right\}$. From the resulting state, we have the following path: $\operatorname{path}_{\mathcal{A}}([\top, 0,0])=\left\{\left(\left\langle\right.\right.\right.$ obs_heating, $\left.\left.\left.\operatorname{tau}_{6}\right\rangle,[\top, 0,1]\right)\right\}$. At this point, we have a non-deterministic choice whether to continue heating, or pour the coffee. $\left.\operatorname{path}_{\mathcal{A}}([\mathrm{T}, 0,1])\right)=\left\{\left(\left\langle\right.\right.\right.$ obs_heating, $\left.\left.\operatorname{tau}_{6}\right\rangle,[\mathrm{\top}, 0,2]\right),\left\langle\right.$ obs_pouring, $\left.\left.\left.\operatorname{tau}_{4}, \operatorname{tau}_{5}\right\rangle,[\perp, 0,1]\right)\right\}$

\subsection{Tests}

Definition 3.3 (Test and Test Suite). A test $\sigma$ for $\mathcal{A}$ is a sequence of pairs $\left\langle\left(\epsilon, o_{0}\right)\right.$, $\left.\left(\ell_{1}, o_{1}\right), \ldots,\left(\ell_{n}, o_{n}\right)\right\rangle$ of type labels $_{v} \times \mathcal{P}^{\text {labels }_{v}}$, where labels $_{v}$ is the set of all visible labels in $\mathcal{A}$. The first component of a pair is a controllable or observable action-label. The second component of a pair is a set of observable labels. A test suite for $\mathcal{A}$ is a set of tests for $\mathcal{A}$.

Given a test $\sigma$, we use $\sigma[i]$ to refer to the $i$ th pair in $\sigma$. Further, we use $\sigma[i]$.execlabel to denote the first component of the pair and $\sigma[i]$.obslabels to denote the second component of the pair. We use $\sigma$.execlabel and $\sigma$.obslabels as the projections of the sequence of pairs in $\sigma$ to a sequence of the first and second components of the pairs, respectively.

Definition 3.4 (Test Execution). Tests are supposed to be executed on an SUT by some tester. The sequence $\sigma$.execlabel represents the sequence of stimuli provided by the tester and feedback observed from the SUT. Thus, this sequence can be viewed as test input and test oracle at the same time. The sequence $\sigma$.obslabels represents a sequence of additional oracles, fixing which observable feedback from the system is in accordance with the model, after a given sequence of actions has been performed or observed. Let us consider the pair $\left(\ell_{1},\left\{\ell_{1}^{\prime}, \ldots, \ell_{m}^{\prime}\right\}\right)$ that is some component of some test. The semantics of such a pair is that $\ell_{1}$ either represents the next stimuli to provide to the system, if $\ell_{1}$ is controllable, or which next observable feedback to expect from the system, to be in accordance with the test. The set of labels $\left\{\ell_{1}^{\prime}, \ldots, \ell_{m}^{\prime}\right\}$ represents all possible observable feedback from the system that is allowed to occur by the model immediately after $\ell_{1}$ was performed or observed. If $\ell_{1}$ is observable, by definition, then it is in the obslabels set of the preceding element of the sequence. The special pair $\left(\epsilon, o_{0}\right)$ is at the beginning of every test, where $o_{0}$ are the observables allowed in the initial state $s_{l}$.

When a test is executed, there can be different outcomes, called test verdicts.

Definition 3.5 (Test Verdicts). We distinguish between three possible test verdicts pass, fail, and inconclusive. If the sequence of actions represented by $\sigma$.execlabel can be fully performed by the tester on the SUT, i.e., controllable stimuli can be provided and observable feedack is observed in the correct sequence, then the verdict is pass. If a partial sequence of actions of $\sigma$.execlabel can be performed up to label $\ell_{i}$, and the SUT provides observable feedback corresponding to an action-label $\ell \in o_{i}$ with $\ell \neq \ell_{i+1}$, then the verdict is inconclusive. Otherwise, the verdict is fail.

The role of the inconclusive verdict is to deal with non-determinism in the internal or observable behavior of the model. The internal state and following behavior of a SUT after providing observable feedback that leads to an inconclusive verdict might be very different from the one that is expected when the test sequence was followed. Therefore, the rest of the sequence cannot be used to determine passing or failing of the test run. The alternative to using inconclusive verdicts are fully adaptive tests, specifying correct test executions for every possible way to resolve 
non-determinism. Such tests have the shape of a tree or a directed acyclic graph, rather than a sequence. However, adaptive tests are much more expensive to create and maintain, since they essentially need to explicitly enumerate all possible behaviors of the system. Therefore, we do not consider such tests in this work.

Example 3.6. A valid test for our example in Figure 1 is $\sigma=\left\langle(\epsilon, \emptyset),\left(\operatorname{ctr} \_c o f f e e,\{\right.\right.$ obs_heating $\left.\}\right)$, (obs_heating, \{obs_heating, obs_pouring\}), (obs_heating, \{obs_pouring\}), (obs_pouring, $\emptyset)\rangle$. Since after producing one coffee, the machine is not dirty enough for cleaning, obs_pouring cannot be followed by an observable. The only possible next action is the controllable ctr_coffee, therefore the set of possible observables after obs_pouring is empty. Suppose we execute that test on some SUT. The sequences of action labels 〈ctr_coffee, obs_heating, obs_heating, obs_pouring has verdict pass, 〈ctr_coffee, obs_heating, obs_pouring〉 has verdict inconclusive, 〈ctr_coffee, obs_pouring $\rangle$ has verdict fail.

\section{MODEL-BASED MUTATION TESTING OF REACTIVE SYSTEMS}

The mutation score [30] is a test suite metric that quantifies the fault detection capability of a test suite. Mutation score is defined as the number of killed mutants divided by the number of created mutants. Its benefit is that it quantifies semantic quality features of implementations, i.e., fault robustness, as opposed to syntactic quality features, such as transition, state, or branch coverage metrics. Its main drawback is the cost associated with creating and analyzing test suites with high mutation score. This work shows a method to obtain the benefits and mitigate the drawbacks. In the following, we define mutants of action systems and define killing of mutants in our setting.

\subsection{Mutated Models}

We study the differences between mutated action systems and their original versions. A mutated action system is a duplicate of a given action system, with the exception of small syntactically correct variations, i.e., mutations. We allow expressions used in assignments and guarded commands to be mutated and assign a unique integer mutation id to every mutation introduced. For an action $\ell: A$ that is an assignment or a guarded command, we denote by $m(\ell)$ the set of mutation ids applied to action $A$. We call a mutation reachable, if its action is reachable. A valid mutation replaces an operator in an expression with any operator that is syntactically allowed. The chosen mutation operators are those standard operators from the literature [30] that are applicable to our modeling formalism. A mutated action system $\mathcal{A}^{m}$ is derived from the original action system $\mathcal{A}$ by inserting exactly one mutation $m$. The general goal of mutation testing is to kill mutants. Roughly speaking, a mutant is killed by a test case if it reveals different execution behavior to its original counterpart.

Example 4.1. A simple mutation in our example from Figure 1 would be to change the guard of obs_cleaning from dirty $\geq 4$ to dirty $<4$. The resulting model is stuck in an endless cleaning loop without ever serving coffee.

\subsection{Classic Strong and Weak Killing}

Intuitively, a mutant is killed by a test if executing that test on the mutated system produces a different result than executing it on the original. What exactly is meant by different result depends on the kind of systems analyzed, as well as the type of the kill. In the realm of mutation testing, there exist various types of mutant kills, most prominently weak and strong kills. A mutant is weakly killed [27] if it produces a different intermediate result for some component of the system. Typically, these components are assumed to simply be the states of the system. A mutant is strongly killed [13] if it produces a different output than its original version. 
Historically, both killing concepts were defined for sequential programs and with respect to a given reference implementation. For example, we could have some function $f$ that takes one input value and returns a value. A mutant could be any syntactic modification of the code defining $f$. A test would be one input value for the function. The test strongly kills the mutant if the function returned a different value for the test argument. It weakly kills the mutant if any intermediate state (e.g., a local variable) of the function differs from the respective intermediate state of the original.

\subsection{Input Output Conformance Killing}

Classic killing concepts do not sufficiently capture the fault detection capability in our setting.

First, in contrast to straight line programs considered in classic mutation analysis, we study reactive systems, for which there are no single points of input and output comparison; rather, inputs and outputs are intertwined.

Second, we perform model-based testing. Thus, in contrast to classic mutation testing, we do not aim to reveal errors in the artifact at hand, i.e., the original model, which we assume to be correct by design, but in systems that supposedly implement the model. A test that passes on a mutated model, but fails on the original model might still pass on an implementation of the original model, because the implementation is allowed to implement more behavior than the model (underspecification is allowed in the model). Therefore, we focus on tests that pass on the original model and fail on a mutated model.

Third, from a testing perspective, it is hard or impossible to witness the inability of a system to provide some observable feedback. In contrast, it is simple to compare a given sequence of stimuli and observations to a test, as described above, and check whether the SUT behaves in accordance with the test. Therefore, we aim to create tests that fail on non-conformant implementations in an observable way, i.e., systems that implement a mutated model provide some additional observable feedback that would not be possible if the system implemented the original model.

Finally, models are often under-specifications of acceptable behavior. This is typically expressed via non-determinism, which needs to be taken into account by our killing analysis.

Formal conformance testing [49] provides a framework that enables us to incorporate all these requirements into mutation killing analysis. The framework was designed to formalize the question whether a given system is correct with respect to a specification. Input output conformance (IOCO) [50] is a conformance relation that is particularly well suited towards our needs. IOCO allows non-deterministic specifications and operates over sequences of input/output transitions. In addition, it treats implementations and specifications in an asymmetric way, which captures the desire to neglect tests that fail on the original, but pass on the mutated model. In our work, mutants take on the role of implementations, whereas the original model takes the role of the specification. For a detailed description of IOCO and its manifold variants, we refer the reader to Reference [50]. Here we only reproduce the essential definitions of IOCO.

Input/Output Transition Systems. IOCO is defined on Input/Output Transition Systems (IOTS). An IOTS is a 5-tuple $M=\left(V, L_{i}, L_{o}, T, v_{l}\right)$, where $V$ is a set of vertices, $L_{i}$ and $L_{o}$ are sets of input and output labels, respectively; $T \subseteq V \times\left(L_{i} \cup L_{o} \cup\{\tau\}\right) \times V$ is a transition relation; and $v_{\iota} \in V$ is the initial vertex.

An action system $\mathcal{A}$ with initial state $s_{\iota}$ can be represented as an IOTS as follows:

$$
v_{\iota}=v_{s_{l}} \text {. }
$$

The set of vertices corresponds to the set of reachable states and extra vertices that represent intermediate states that occur during successor computation,

$$
V=\left\{v_{s} \mid s \text { is a reachable state of } \mathcal{A}\right\} \cup\left\{v_{i}^{\pi}\left|i \in[1, n-1], \pi \in \operatorname{path}_{\mathcal{A}}(s),\right| \pi . l \mid=n\right\} .
$$


The sets $L_{i}$ and $L_{o}$ are the sets of all controllable and observable labels, respectively,

$$
\begin{aligned}
& L_{i}=\{\ell \mid \ell \text { is a controllable action label of } \mathcal{A}\} \\
& L_{o}=\{\ell \mid \ell \text { is an observable action label of } \mathcal{A}\} .
\end{aligned}
$$

The transitions correspond to the successor paths, as defined in the semantics of action systems. We define them via intermediate sets of transitions $T_{s}$ that encode the outgoing transitions for a reachable state $s$,

$$
\begin{gathered}
T=\bigcup_{s \text { is a reachable state of } \mathcal{A}} T_{s}, \text { where } \\
T_{s}=\left\{\left\{\left(v_{0}, a_{0}, v_{1}\right), \ldots,\left(v_{n-1}, a_{n-1}, v_{n}\right)\right\} \mid \pi \in \operatorname{path}_{\mathcal{A}}(s), v_{0}=v_{s}, v_{n}=v_{\pi . s}, v_{i}=v_{i}^{\pi} \text { for } i \in[1, n-1],\right. \\
\left.a_{i}=\pi . l[i] \text { if } \pi . l[i] \in L_{i} \cup L_{o}, a_{i}=\tau \text { otherwise }\right\} .
\end{gathered}
$$

While $v_{0}$ and $v_{n}$ are vertices that correspond to a reachable state of $\mathcal{A}, v_{1} \ldots v_{n-1}$ are fresh vertices. These are not associated with a specific state of $\mathcal{A}$.

We denote by $\mathcal{A}$ both the action system and IOTS representation of the same model. To compare states of IOTS representations, we define a function states $(U)$ that for a set of vertices $U$ returns the set of all states of $\mathcal{A}$ that have a corresponding vertex in $U$.

Note that the IOTS representation is defined via the set of reachable states and their successor paths. Since in our setting these sets are not known a priori, we build the IOTS representation during model exploration by maintaining a partial IOTS, which is initialized by the empty IOTS, to which we continuously add explored successor paths.

IOCO Killing. We define $v \stackrel{a}{\longrightarrow} v^{\prime}$ as one step in the IOTS: $\left(v, a, v^{\prime}\right) \in T$. This is extended to sequences of symbols $\varsigma$ in the obvious way and denoted as $v \stackrel{\varsigma}{=} v^{\prime}$. The sequence $\varsigma$ does not contain $\tau$-transitions, which $\stackrel{S}{\Longrightarrow}$ may execute in between symbols. This introduces a non-determinism and for a fixed $v, v \stackrel{S}{\Longrightarrow} v^{\prime}$ may be valid for multiple $v^{\prime}$. We introduce $v$ after $\varsigma$ to mean all vertices reachable from $v$ after sequence $\varsigma:\left\{v^{\prime} \mid v \stackrel{\varsigma}{\Longrightarrow} v^{\prime}\right\}$. Instead of $v$ after $\varsigma$, we may also write $M$ after $\varsigma$ for some IOTS $M$ to mean $v_{\iota}$ after $\varsigma$ with $v_{\iota}$ being the initial vertex of $M$. In addition to observable and controllable behavior, IOCO takes into account the inability to produce output via quiescence. To this end, a IOTS $M_{\delta}$ is created, by adding to every vertex of $M$ that has no outgoing internal or observable label, a self-loop with a special label $\delta$. All non-blocking traces (i.e., traces not ending in an abort statement) of $M_{\delta}$ are called Straces $(M)$. We use out(v) to denote the set of all output labels on outgoing transitions from $v$. If $v$ is a quiescent vertex, then the set also contains $\delta$. The definition of out is extended to sets of vertices by taking the union of the results. Finally, the definition of IOCO conformance of implementation $I$ and model $M$ is

$$
\text { I ioco } M=\forall \varsigma \in \operatorname{Straces}(M): \operatorname{out}(I \text { after } \varsigma) \subseteq \operatorname{out}(M \text { after } \varsigma) .
$$

This definition intuitively ensures two things: (a) The implementation $I$ must not produce an output that the model $M$ cannot produce. The implementation may, however, accept additional inputs, meaning that it can offer additional functionality. (b) The implementation may not stay quiet (do nothing) when the model can give an output. This ensures that the trivial implementation, that simply does nothing, does not conform to the specification.

Definition 4.2 (Weak Killability). $\mathcal{A}^{m}$ is weakly killable if

$$
\exists_{\varsigma} \in \operatorname{Straces}(\mathcal{A}) \text {. states }\left(\mathcal{A}^{m} \text { after } \varsigma\right) \nsubseteq \text { states }(\mathcal{A} \text { after } \varsigma) .
$$

Definition 4.3 (Strong Killability). $\mathcal{A}^{m}$ is strongly killable if not $\left(\mathcal{A}^{m}\right.$ ioco $\left.\mathcal{A}\right)$. 
Tests are witnesses to the fact that a mutated action system is weakly or strongly killable. The definitions of weak and strong killability immediately give us sequences of labels that are such witnesses. Since tests by construction do not end in abort statements, the only difference between $\varsigma \in \operatorname{Straces}(\mathcal{A})$ and the execlabel component of tests are additional $\delta$ labeled transitions. However, since tests hold all possible following observables in the obslabels component, we can easily add $\delta$ labeled transitions whenever the obslabels component is empty. Given a test $\sigma$, we denote by Strace $(\sigma$.execlabel) the sequence of action labels obtained in this way.

Definition 4.4 (Weak Kill). A test $\sigma$ for $\mathcal{A}$ weakly kills $\mathcal{A}^{m}$ if $\operatorname{states}\left(\mathcal{A}^{m}\right.$ after $\operatorname{Strace}(\sigma$.execlabel $\left.)\right) \nsubseteq \operatorname{states}(\mathcal{A}$ after Strace $(\sigma$. execlabel $))$.

Definition 4.5 (Strong Kill). A test $\sigma$ for $\mathcal{A}$ strongly kills $\mathcal{A}^{m}$ if $\operatorname{out}\left(\mathcal{A}^{m}\right.$ after Strace $(\sigma$.execlabel $\left.)\right) \nsubseteq$ out $(\mathcal{A}$ after Strace $(\sigma$. execlabel $))$.

Note that if a test $\sigma$ strongly kills an action system $\mathcal{A}^{m}$, then there is a test execution of $\sigma$ on $\mathcal{A}^{m}$ that fails. This failing execution is given by the sequence leading to the unexpected observable. Furthermore, if some SUT implements $\mathcal{A}^{m}$, then the test also fails on this implementation.

Example 4.6. Consider a mutant that changes the guard of obs_cleaning from dirty $\geq 4$ to dirty $\geq 3$. The mutant cannot be strongly killed, because dirty is always incremented by 2 . It cannot be weakly killed, because the internal state never differs from the original. Consider a mutant that changes the assignment in $\mathrm{tau}_{4}$ from dirty $\leftarrow$ dirty +2 to dirty $\leftarrow$ dirty +3 . This mutant can be weakly killed. The state after one ctr_coffee is $[\perp, 3,1]$ instead of $[\perp, 2,1]$. However, there is no observable difference in behavior of the model, because it would still clean after two coffees. Finally, consider a mutant that changes the effect of $\operatorname{tau}_{4}$ from dirty $\leftarrow$ dirty +2 to dirty $\leftarrow$ dirty +4 . This effectively means that the machine cleans itself after every coffee. This mutation is strongly killed by any test that requests two coffees, since the original model pours the second coffee, while the mutated model cleans.

\section{STATE SPACE EXPLORATION}

In this section, we describe our main algorithm. The algorithm searches for mutants using heuristic guided branching search and performs killing analysis.

The main source of inspiration for the search procedure was the RRT path planning algorithm [36]. An interesting property of RRT for our problem is that it is able to reach different areas of large state spaces quickly [34]. We assume that in different areas of the state space different actions are enabled and as a consequence different mutations can be found. RRT splits the search into small, independent sub-searches, which we call branching search. The (almost) independence of sub-searches allows branching search to be implemented in a parallel fashion. In our algorithm description, we refer to sub-searches as tasks and denote them by task. Each task starts in some previously discovered state and explores the state space for a fixed number of steps. Over the course of the search, we repeatedly create new tasks. Where a task starts and which transition it takes is determined by heuristics.

The heuristics of classic RRT are distance based and have been applied in domains, such as motion planning [6], or well-defined discrete planning problems [11], where distance metrics naturally arise. On top of using different distance metrics, alternations in the way RRT is used typically target the structure of the search algorithm itself, such as DRRT [21] for dynamically adjusting to changing environments or T-RRT [28] for continuous cost optimization. We found the distance metric that naturally arises for our models, the Hamming Distance, does not always provide satisfactory results, while the search skeleton indeed works well for us. Therefore, we 
experimented with different heuristics to replace distance-based decisions, using the same overall search procedure.

Throughout the section, we assume a fixed action system $\mathcal{A}$ with variables $\mathcal{V}$ and initial state $s_{l}$.

\subsection{Search Algorithm}

Algorithm 1 shows the pseudo code of our search algorithm. The algorithm has the following parameters that need to be instantiated: BRANCHLENGTH is the number of steps performed by each single task. MAXSTEPS is the total number of states explored by all tasks combined. To explore a state $s$, we calculate the predicate $\operatorname{path}_{\mathcal{A}}(s)$, i.e., compute all its outgoing transitions and successor states. CREATETASK is the heuristic to create new tasks, which essentially chooses the starting state of tasks, and possibly creates a goal for the task. SelectSuccessor is the heuristic to select which successor state should be explored next by the task. Every task task is represented as a tuple consisting of its current state task.state, its goal states task.goal, which is used by some successor state heuristics, and the number of steps it already performed task.steps.

The algorithm executes two main blocks of instructions in a loop until the maximum number of steps has been performed. The first block (lines 7-9) starts new tasks. We want to start a new task initially (stepCount $=0$ ) and whenever the number of steps between the current and the last time a task was created exceeds the maximal number of steps for each task divided by 4 . We delay the creation of new tasks as opposed to eagerly starting as many tasks as possible, because start states of tasks are chosen among the previously discovered states. Therefore, starting many tasks at once would result in all of them starting in the same state, which is not the branching behavior we typically want. We found BRANCHLENGTH divided by 4 to be a good compromise between starting enough tasks to leverage branching search and its feature to parallelize the search and delayed starting to benefit from prior exploration. When creating a new task, we heuristically choose a start state among all states in the visited set and a goal state. Note that when a task reaches its goal state, the task does not stop, but explores successor states close to its goal state. Alternatively, the task could be terminated and a new task could be started.

The second block (lines 10-26) performs the essential part of the search, computing successors, making the decision which successor to explore next, and performing killchecks of mutants. To compute successors, that is to evaluate $\operatorname{path}_{\mathcal{A}}(s)$, the underlying do-od loop of the model is executed, guards are evaluated, and assignments are applied to the state. Across the whole testcase generation approach this is by far the computationally most expensive part. Therefore, it is beneficial to parallelize this part of the search.

All successor states are added to the visited set of states for possible further exploration and the current state is added to the explored set of states. In order not to redo work, the SELECTSUCCESSOR heuristic may only choose successor states that are not already in the explored set. We do not gain anything from re-exploring previously explored states, therefore if possible we choose successor states only among those that were previously unexplored. If all successor states were already previously explored, then we pick a successor state at random. Alternatively the task could be aborted. However, this approach has the a danger of getting stuck, if tasks are repeatedly started in states for which all successor states are explored.

We add every mutation that is reached on one of the successor paths to the set of reached mutations. Finally, we increase the task-specific as well as the global step count by one.

\subsection{Killcheck}

The strong killcheck explores the mutated model from task.state for a given number of steps $n$ and compares the results to the matching exploration results of the original model. Its goal is to 


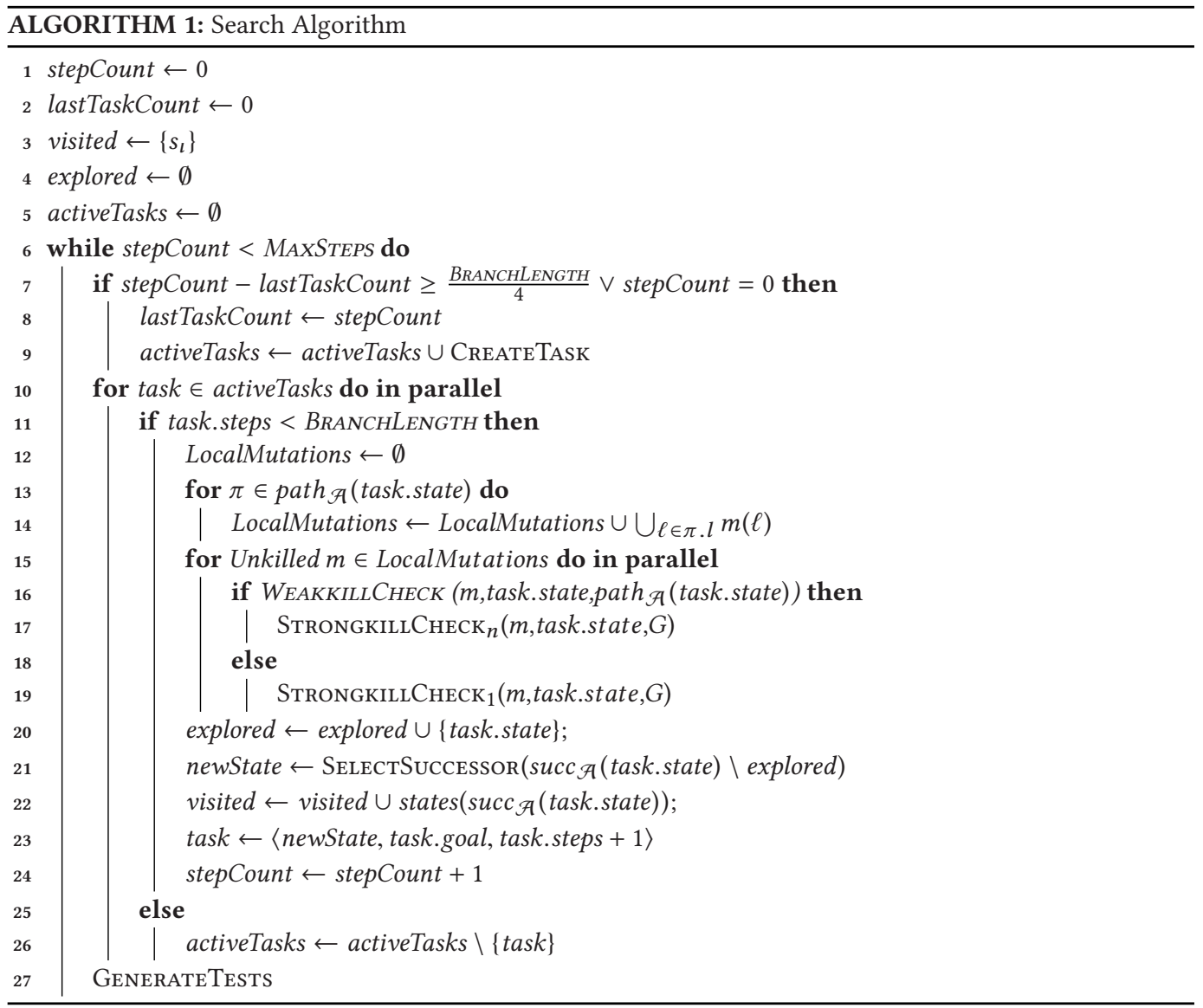

show observable differences according to Definition 4.3. The mutant may only show an observable difference after an unknown number of steps.

Note that task.state is a reachable state in the mutant $\mathcal{A}^{m}$. Since we only analyse previously unkilled mutants, the mutant is not weakly killed along the path leading to task.state. Therefore, there is a sequence of states from $s_{l}$ to task.state that is shared by $\mathcal{A}$ and $\mathcal{A}^{m}$. Starting the killcheck only from task.state is a key insight to lower computational the costs of the killing analysis.

The weak killcheck simply checks whether the mutant can reach a successor state $s$ where $\exists s^{\prime} \in$ $\operatorname{succ}_{\mathcal{A}^{m}}(s) . s^{\prime} \notin \operatorname{succ}_{\mathcal{A}}(s)$. We do not generate test cases for weak kills, but the weak kill is used to decide whether a strong killcheck should be performed for $n>1$. A weak kill is a precondition to possibly finding a strong kill by propagating the introduced difference, because if the state does not diverge after executing the mutant there will be no change in observable behavior further on. That means that STRONGKILlCHECK $n$ for $n>1$ makes sense only after a weak kill. However, note that the mutation may enable an observable action that does not change the state. That is, we can have a strong kill without a weak kill. Therefore, Algorithm 1 performs STRONGKILLCHECK 1 in line 19, even when no weak-kill is detected. The parameter $n$ needs to be selected upfront. Higher values incur higher computation costs.

Example 5.1. In our running example, suppose we are at state $s=[\top, 0,1]$. We have that $\operatorname{path}_{\mathcal{A}}(s)$ consists of the pairs (〈obs_pouring, $\left.\operatorname{tau}_{4}, \operatorname{tau}_{5}\right\rangle,[\perp, 2,1]$ ) and (〈obs_heating, $\left.\operatorname{tau}_{6}\right\rangle$, $[\perp, 0,2])$. 
Table 2. Heuristics for CREATETASK

\begin{tabular}{ll}
\hline Name & Description \\
\hline Init & $\begin{array}{l}\text { goal } \leftarrow \operatorname{rand}(\mathcal{S}) \\
\text { start } \leftarrow s_{l}\end{array}$ \\
\hline RandCT & $\begin{array}{l}\text { goal } \leftarrow \operatorname{rand}(\mathcal{S}) \\
\text { start } \leftarrow \operatorname{rand}(\text { visited })\end{array}$ \\
\hline RGoal & goal $\leftarrow \operatorname{rand}(\mathcal{S})$ \\
& start $\leftarrow \arg \min _{s \in \text { visited }}(d(s$, goal $))$ \\
\hline CGoal & goal $\leftarrow \operatorname{combine}($ visited $)$ \\
& start $\leftarrow \arg \min _{s \in \text { visited }}(d(s$, goal $))$ \\
\hline PGoal & start $\leftarrow \operatorname{rand}(\mathcal{U} / \mathcal{R})$ \\
$(\mathcal{U} / \mathcal{R})$ & goal $\leftarrow \operatorname{perturb}$ start \\
\hline RoRoCT & Round robin of other heuristics \\
\hline
\end{tabular}

Table 3. Heuristics for SelectSuccessor(states)

\begin{tabular}{ll}
\hline Name & Description \\
\hline RandSS & $s^{\prime}=$ rand $($ states $)$ \\
\hline Dist & $\Lambda=\{(t, \lambda) \mid t \in$ states, \\
& \multicolumn{1}{c}{$\lambda=d(t$, task.goal $)\}$} \\
& $s^{\prime}=\operatorname{select}(\Lambda)$ \\
\hline Part & $\Lambda=\{(t, \lambda) \mid t \in$ states,$\lambda=\alpha(t)\}$ \\
& $s^{\prime}=$ select $(\Lambda)$ \\
\hline LocalBFS & if max depth not reached \\
& add all $s \in$ states $\backslash$ explored to queue \\
& $s^{\prime}=$ queue.pop \\
\hline RoRoSS & Round robin of other heuristics \\
\hline
\end{tabular}

The mutant that changes the effect of $\operatorname{tau}_{4}$ from dirty $\leftarrow$ dirty +2 to dirty $\leftarrow$ dirty +4 is reached in $\operatorname{path}_{\mathcal{A}}(s)$. However, it is not strongly killed in one step, since it does not diverge from the original yet. But since the state is different, we would keep exploring further to find the observable difference in the next step, where a cleaning would take place in the mutant but not in the original.

\subsection{Distance Metrics}

Many of our heuristics use distance metrics to guide the search. The notion of distance is also a key concept of rapidly exploring random trees. RRTs are usually applied to path planning in the plane, where the notion of distance is naturally given by the Euclidean distance.

We use the Hamming Distance, a distance metric that defines distance 0 for equal values and distance 1 for unequal values. We calculate the distance between two lists as the average number of elements differing. We calculate the distance between two states as the average Hamming Distance between all its variables. Note that two states always have the same variables as their number is fixed by the action system. The intuition is that most actions manipulate only a small subset of the variables and executing the action that sets most of the variables to the desired value is beneficial to reaching our goal.

\subsection{CreateTask Heuristics}

CREATETASK heuristics create fresh tasks by picking a start state from the previously visited states visited, and a goal state from the set of all possible states. The fresh task is the tuple $\langle$ start, goal, 0$\rangle$.

Table 2 provides an overview of the CREATETASK heuristics described in more detail below. We assume a function $\operatorname{rand}($.) that returns a uniformly random element of its input set. $\mathcal{S}$ denotes the set of all possible states. That is, if we have a system with two integers with values between 0 and $10, \mathcal{S}$ is the set $[0,10] \times[0,10]$. It is not guaranteed that all of these states are reachable. For list variables, we first create a random list length and then create random values recursively.

Init. The Init heuristic always chooses the initial state as the start state. Therefore, it is more likely to make different choices on early branches in the action system. Its downside is that is might not penetrate the model as deeply as other heuristics and might repeat work previously performed when there are no unexplored options close to the initial state. The goal state is simply chosen randomly among all possible states.

RandCT. The RandCT heuristic simply chooses one random state in visited. The goal state is chosen randomly among all possible states. 
RGoal. The RGoal heuristic resembles classic RRT search. A goal state is chosen randomly among all possible states. The start state is then simply the closest state in visited according to distance $d$.

CGoal. The CGoal heuristic works similar to RGoal. However, a goal state is not chosen at random, but is created as a random combination of previously visited states. For every variable in the model, a value is picked from a randomly selected state of visited. The idea of this heuristic is to create goal states that are more likely to be reachable than purely random states.

PGoal. The PGoal heuristic exists in two flavors called PGoal $(\mathcal{U})$ and PGoal $(\mathcal{R})$. Both have in common that they turn around the selection process used by RRT by first picking a start state and afterwards producing the goal from that state. The state to start from is randomly chosen from the set of unique states $\mathcal{U}$ or rare value states $\mathcal{R}$. These sets are defined as follows: $\mathcal{U}=\{s \in$ visited $\mid s$ was inserted into visited at most once $\}, \mathcal{R}=\{s \in$ visited $\mid \exists v \in \mathcal{V}$ such that $\mid\{t \in$ visited $\mid$ variable $v$ has the same value in $s$ and $t\}|<|$ visited $\mid / 10\}$. After picking a start state, a goal state is created by perturbing a fraction of the variable values of the chosen start state. By perturbing, we mean modifying a value, either by selecting a different value at random or choosing integer values close to original integer values. The idea of these heuristics is to explore regions of the state space that have yet been sparsely explored.

RoRoCT. The RoRoCT heuristic is a meta-heuristic that creates tasks according to multiple CREATETASK heuristics in a round-robin fashion. For the experiments presented in this article, RoRoCT chooses among all other heuristics. The idea of round robin is to combine the strengths of multiple heuristics. If one task gets stuck due to a bad heuristic decision, then the next one is not likely to get stuck in the same way.

\subsection{SelectSuccessor Heuristics}

SELECTSUCCESSOR heuristics choose one state out of a given set of states to explore next. Table 3 shows the different heuristics and gives a short overview.

We assume the existence of a helper selection function select(.) that picks one element out of a set of states and according to provided state evaluations. As instantiations of this function, we experimented with greedy, simply taking the best evaluation; weighted, picking probabilistically according to the distribution given by the evaluations; and bucket, which first groups states into buckets of equal evaluation, then picks a bucket probabilistically according to the distribution given by the evaluations, and, finally, picks a state randomly within the bucket.

RandSS. The Rand heuristic randomly picks a state in states.

Dist. The Dist heuristic assigns to each state in states its distance to the task's goal state and picks a successor state via one of the above select (.) functions. This heuristic emulates classic RRT search.

Part. The Part heuristic assigns to each state in states the new object states evaluation $\alpha($.$) and$ picks a successor state via one of the above select(.) functions. $\alpha(s)$ returns the number of object values that occur in $s$, but not in the global set of previously visited states visited. An object value is the projection of the state to the values of one particular object instance. When checking whether we have encountered an object value before, we check whether that particular object value has been encountered in any instance of the object's class before.

The idea of this heuristic is to have a fine grained characterization of new information in states. For large models, the search algorithm constantly finds new states, since the number of combinations of variable valuations is high. Objects of the same type typically have a symmetric role, in the sense that it does not matter which particular instance is part of a state that might trigger 
new behavior. The Part heuristic exactly tries to capture and quantify the novelty of information a state supplies.

LocalBFS. The LocalBFS heuristic works a bit different than the other heuristics. The idea of this heuristic is to combine heuristic-driven exploration with complete local search via depth bounded breadth-first search. Heuristic-driven exploration is supposed to quickly expand the search space into diverse areas, while bounded breadth-first search is supposed to uncover rare, bottleneck transitions that open up new regions of the state space. Since many of our target models are too large for full breadth-first search, we limit the depth bound of local BFS searches to a fixed bound. Furthermore, to balance between heuristic-driven exploration and complete local search, we perform local BFS searches with a given frequency, e.g., every fourth task performs bounded BFS, while the others perform distance-based exploration.

RoRoSS. The RoRoSS heuristic is a meta-heuristic that selects successors according to multiple SELECTSuCCESSOR heuristics in a round-robin fashion. The heuristic is fixed for one task for its whole lifetime. Similarly to RoRoCT for task creation, we want to benefit from the advantages of all other heuristics and avoid to get stuck during the search.

\subsection{Full Breadth-First Search}

Using our search framework, we can also instantiate the heuristics to perform full breadth-first search, exploring all reachable states in the process. This strategy is only viable for small models. However, on models that are small enough to be explored fully, it produces interesting insights. For example, we are now able to determine the state space and the number of reachable mutants. These measurements serve as a baseline for comparison of heuristics.

To perform full breadth-first search in our framework, in addition to the data kept in Algorithm 1, we maintain a queue of states. Tasks add successor states to this queue. The condition to create new tasks is replaced by a check whether there are still unexplored states in the queue. The CREATETASK heuristic simply picks the first unexplored state of the queue. Finally, branch length is set to 1, therefore the SELECTSUCCESSOR does not have an effect. The difference to LocalBFS is that full breadth-first search operates not on the task heuristic level, but on a global search level. That is, multiple tasks correspond to one full breadth-first search, whereas only one single task corresponds to one LocalBFS search. Furthermore, with full BFS we do not mix the exploration with other heuristics. Instantiating our algorithmic framework this way allows us to use the parallelization framework for breadth-first search.

\subsection{Test-case Generation}

In this section, we show how we transform findings made during exploration and killchecking into tests. To this end, we assume that during the exploration of $\mathcal{A}$ we built up a partial IOTS iteratively. We do this by adding transitions with controllable or observable action labels corresponding to paths returned by $\operatorname{path}_{\mathcal{A}}(s)$. In addition, for every vertex $v$ that we add to the IOTS, we record the full set of outgoing transitions with observable labels out $(v)$. Since there might be paths in $\operatorname{path}_{\mathcal{A}}(s)$ that contain only internal action labels, it can be necessary to recursively call path $h_{\mathcal{A}}(s)$. We associate states of $\mathcal{A}$ to vertices of its partial IOTS, essentially re-using vertices for states that are re-discovered during the search. Therefore, the resulting IOTS may have cycles.

In the case where we find a strong kill for some mutant $m$, we annotate the edge $e_{m}$ resulting in the kill with mutant $m$. Since we do not attempt to kill previously killed mutants again, we annotate at most one edge per mutant. To create a test $\sigma$ from the resulting IOTS that kills $m$, we first need to find a path from the initial vertex that includes $e_{m}$. This path is $\sigma$.execlabel and by collecting outgoing transitions with observable labels along the path, we obtain $\sigma$.obslabels. We 
Table 4. Properties of the Test Models

\begin{tabular}{lrrrrrrrrr}
\hline Model & Source & Obj & Ins & $\boldsymbol{\ell}$ - Act & Vars & States & S Size & Traces & LoC \\
\hline ChassisDynoDSL & DSL & 1 & 9 & 5 & 6 & 14 & 0.02 & 162 & 124 \\
PartCountDSL & DSL & 1 & 19 & 7 & 11 & 222 & 0.03 & $1 \cdot 10^{3}$ & 391 \\
DemoTopology & Event-B & 1 & 120 & 9 & 25 & TO & 4.9 & $1 \cdot 10^{3}$ & 451 \\
AlarmSystem & UML & 3 & 4 & 53 & 42 & 76 & 0.3 & $55 \cdot 10^{6}$ & 967 \\
Debounce & UML & 3 & 30 & 34 & 29 & 560 & 0.1 & $52 \cdot 10^{0}$ & 655 \\
PartCountUML & UML & 3 & 18 & 103 & 55 & 12138 & 0.5 & $41 \cdot 10^{3}$ & 1834 \\
Defibrillator & UML & 4 & 17 & 140 & 67 & TO & 0.4 & $3 \cdot 10^{3}$ & 2949 \\
Loader & UML & 4 & 1692 & 105 & 98 & MO & 0.8 & $108 \cdot 10^{9}$ & 2009 \\
MMS & UML & 125 & 86 & 1023 & 1490 & MO & 8.2 & $2 \cdot 10^{3}$ & 8281 \\
LBT & UML & 2373 & 826 & 1763 & 27959 & MO & 182.4 & $51 \cdot 10^{3}$ & 33289 \\
\hline
\end{tabular}

propose two methods to construct paths to kills. Both methods implicitly make sure not to get stuck in cycles, by preventing to traverse any transition more than once.

Greedy. We can traverse the graph greedily in a left to right depth first search manner. During the traversal, we maintain paths corresponding to the transitions we traversed. As soon as we encounter a transition corresponding to a kill of mutant $m$, we store that path for $m$. Greedy is the simplest version of traversing the graph to construct the paths with kills. It only needs one traversal iteration through the graph and needs $O(|V|)$ time, as well as $O(|V| \cdot \# M u t a n t s)$ space.

Shortest Paths. We can also traverse the graph first in a breadth-first search manner, annotating vertices with their depth. As soon as we encounter a transition corresponding to a kill of mutant $m$, we traverse the graph backwards, always following the vertex with the lowest depth to construct the shortest paths to the initial vertex $v_{l}$. Alternatively, for each frontier in the BFS search, we can also store the shortest path to the initial vertex $v_{l}$. This approach either needs $O(|V| \cdot \#$ Mutants $)$ time, when traversing the graph towards the initial vertex for each mutant, or $O\left(|V|^{2}\right)$ space in the worst case, when each node stores the shortest path towards the initial vertex.

Our goal is that test suites do not contain redundant tests, in the sense that one test is a prefix of another. While we can easily remove the smaller tests, we chose to avoid producing them in the first place by improving our algorithm. The essential idea is to perform test-case generation only after exploration, prioritize mutants that are killed on transitions with great distance to the initial vertex, and collect other mutants that are also killed by the constructed tests. Due to space limitations, we omit the presentation of the technical details.

\section{MODELS}

Table 4 provides a comparison of some of the key properties of the test models considered in this article. The models were collected from several industrial use cases. The use cases for AlarmSystem, PartCountUML, and Loader are described in References [2-4].

The models were automatically translated to action systems by MoMuT from three different modeling languages: UML, Event-B and a textual domain specific language (DSL). This source modeling language is given in the Source column. In this work, we consider only the resulting action systems. Therefore all properties of the models described here relate to the action system representation. The Obj column shows the number of objects in the action system. Each object has its own independent do-od loop and can act concurrently to all other objects. Although not every object is always allowed to make a transition in every iteration, this property gives an indication of the amount of concurrent actors in the respective model. The Ins column lists the number of inputs 
the action system can accept. For controllable actions with parameters, the Cartesian product of the parameter ranges has been used, over-estimating in some cases. The $\ell$ - Act column shows the number of labeled actions in the action system. The Vars column shows the total number of variables of the action system. The States column shows the result of full BFS in terms of number of states found, if successful, timeout (TO) after 24 hours, or memory out (MO) when having 378GB RAM available. The $\mathbf{S}$ Size column shows the size of a single state given in kilobytes, i.e., the maximally required memory to represent one state of the action system, assuming all lists are of maximal length (except for LBT where we chose to report the minimal length lists as the maximum would have been too much of an overestimation). Unfortunately, we cannot report reachable state space size. We tested the reachable state space for the medium sized MMS and found 2.5 million states before running out of memory. The Traces column shows the number of different execution traces that are possible, considering the non-deterministically composed actions of the do-od blocks. Note that this is a theoretical value as not all traces may be feasible due to guarded commands not being enabled. Finally, the LoC column shows the number of lines of code of the action system model.

We provide a brief introduction of the case studies and references with more information about them when available.

AlarmSystem. AlarmSystem is a simple model of a car alarm system. Previous results with earlier generations of MoMuT and the model have been described in References [2, 4], where coverage numbers are not directly comparable to this work, because the set of mutations has been extended since then.

Debounce-Signal Debouncing Algorithm. Debounce models a debouncing algorithm used in the domain of safety critical industrial control. It is counting time ticks depending on changes of an input value to decide if the changes shall be considered transient, caused either by bounces of a switch contact or by electro magnetic interference.

PartCountUML-Particle Counter (UML). PartCountUML is a model of a remote control protocol of an exhaust measurement device. In terms of complexities posed, the model is slightly more complex than AlarmSystem. Our initial findings of test-case generation for PartCountUML in an industrial context have been published previously [3]. Here, we use the same model with our new test-case generation engine.

PartCountDSL-Particle Counter (DSL). PartCountDSL is derived from a domain specific language (DSL) model reproducing the functionality of PartCountUML. The model translated from the DSL is leaner than the one coming from UML, the resulting action system models are differently structured. Due to this, despite expressing the same functionality, it contributes to the diversity of the models used in the experiments.

ChassisDynoDSL-Chassis Dyno Controller (DSL). ChassisDynoDSL is a very simple second measurement device, written in the same DSL as the model above.

Defibrillator-Automated External Defibrillator. Defibrillator models the diagnostic logic of an automated external defibrillator device.

Loader-Loader Bucket Implement. Loader models the control loop (including user feedback and error handling) of a bucket loader implement controller. The controller receives joystick deflection values as inputs and computes output values that will drive valves controlling the movements of the bucket. Although Loader is a rather small model, it is highly complex, as can be seen in Table 4: Due to heavily parametrized actions, it requires the highest number of traces for one iteration of the do-od block. Initial findings with previous versions of MoMuT and the use case can be found in Reference [4], which reports findings on a partial model of the system. 
MMS, LBT-Railway Interlocking Systems. MMS and LBT are instantiations of a railway interlocking system. The original UML models consist of two parts each: one shared general model that defines all classes and data structures and one that instantiates the objects needed for the station. While MMS represents a minimal station that allows trains to pass one another, LBT is a model of a mid sized, real-life railway station. Its layout comprises 37 track sections, 56 track relays, 34 switches, 22 main signals, and 145 train routes the operator can select from. MMS, in contrast, only comprises 10 track sections, 4 track relays, 2 switches, 6 main signals, and 10 train routes. Both models are highly non-deterministic due to 2373 (LBT), and 125 (MMS) concurrently running objects. The objects are used to model both physical and logical entities, such as train routes. Both models make extensive use of lists and forall/exists quantifiers. For example, LBT includes more than 9000 lists in the state, has more than 50 exists quantifiers, and over 100 forall quantifiers that have a maximum nesting depth of five.

DemoTopology-Interlocking Logic. DemoTopology models generic rules for safe operation of a railway interlocking system. As such, its functionality is a subset of MMS and LBT. The used station layout is slightly more complex than that of MMS. In contrast to the UML-based interlocking system models, this model uses only a single instance and no concurrent objects. The original model is expressed in Event-B [1]. Event-B models make heavy use of sets and maps, which are not supported natively in action systems and have to be emulated using lists. This emulation identifies two lists whose elements are permutations of the other as being unequal, i.e., adding two elements in different order would yield the same state in the Event-B semantics, but in our semantics the resulting states are different. That causes our full BFS exploration to time out. DemoTopology is a variant of the model described in Reference [44].

The UML models have been built by researchers in close cooperation with industry partners for their real use cases. The DSL and Event-B models have been built by industry partners. As can also be seen in Table 4, the models vary substantially in their characteristics, not only in size itself. The selected models provide different challenges for our test-case generator. In terms of complexity, LBT is the biggest example because of its high number of concurrent objects, with MMS following at some distance. Debounce is the simplest but still uses an integer to model discrete time. The latter is used in all models except the railway interlocking ones. Loader's challenging complexity stems from both its use of many concurrent timers and the large allowed value ranges for its input parameters, increasing the state space.

\section{EXPERIMENTS}

\subsection{Implementation}

The algorithms and heuristics presented in this work are implemented in the MoMuT tool [2]. To cope with the larger models, the tool is tailored towards scalability. To this end, the tool is written in $\mathrm{C}++$ and the original and mutated action systems are just-in-time-compiled $[8,35]$ to machine code, which allows us to execute transitions fast and leverage compiler optimizations.

As mentioned in earlier sections, we implemented the search procedure in an asynchronous parallelized way. There is a central scheduler, which accumulates and distributes data and performs the CREATETASK as well as SelectSuccessor computations. Additionally, there is a set of workers that perform the labor intensive job of $\operatorname{path}_{\mathcal{A}}(\cdot)$ computations and kill checking. Workers store gathered data in buffers, which are repeatedly harvested by the central scheduler. Workers request new tasks from the scheduler actively. Therefore, our parallel computation scheme has two synchronization points between scheduler and workers: the harvesting of buffers and obtaining new tasks. In between these synchronization points, all threads run asynchronously. 
Table 5. Experiments Summary

\begin{tabular}{lrrrrrrrr}
\hline Model & Mutants & Reach & SKill & WKill & \# Tests & TestL & Time & Runs \\
ChassisDynoDSL & 184 & 178.9 & 117.8 & 49.1 & 9.6 & 1.2 & 0.6 & 722 \\
PartCountDSL & 589 & 451.4 & 320.4 & 118.0 & 47.7 & 2.9 & 1.9 & 1089 \\
DemoTopology & 171 & 154.4 & 67.7 & 40.9 & 7.4 & 4.5 & 1303.3 & 158 \\
AlarmSystem & 818 & 646.6 & 210.0 & 215.8 & 16.8 & 3.8 & 5.1 & 1122 \\
Debounce & 1015 & 922.5 & 122.9 & 185.6 & 18.8 & 4.8 & 30.5 & 1262 \\
PartCountUML & 2257 & 2144.0 & 711.6 & 437.3 & 74.9 & 225.8 & 201.9 & 1262 \\
Defibrillator & 3186 & 1907.9 & 613.7 & 959.8 & 35.0 & 899.2 & 258.2 & 440 \\
Loader & 3291 & 1995.5 & 1143.0 & 300.3 & 34.7 & 64.2 & 4801.8 & 154 \\
MMS & 6391 & 2686.2 & 885.5 & 994.8 & 39.1 & 88.7 & 1032.1 & 258 \\
LBT & 1884 & 214.1 & 48.5 & 65.6 & 10.9 & 41.9 & 25189.2 & 16 \\
\hline
\end{tabular}

The implementation currently only supports strong killcheck of depth 1 . Higher depth requires more bookkeeping and exploration of the original system in directions governed not by heuristics but by the transitions resulting in weak kills of mutants. Note that for strong killcheck of larger depth, we need only to explore the original system deeper but also every weakly killed mutant. This potentially blows up the search cost. Interestingly, as can be seen below, a considerable amount of mutants can be strongly killed at depth 1 .

\subsection{Experimental Setup}

We used three different machines for our experiments. Machine 1 has an Intel(R) Xeon(R) CPU at 3.47GHz, 24 cores, and 189GB RAM. Machine 2 has an Intel(R) Xeon(R) CPU at 2.80GHz, 40 cores, and 378GB RAM. Machine 3 has an Intel(R) Xeon(R) CPU at 2.00GHz, 60 cores, and 252GB RAM.

We have four parameters in Algorithm 1 that can be instantiated: CreateTask, SelectSuccessor, MaxSteps, and BranchLength. There is a hidden fifth parameter: the random seed. Since a lot of our CreateTask and SelectSuccessor heuristics involve randomness a pseudorandom number generator is used to ensure reproducibility of the results. Furthermore, we can control the maximum amount of cores used for parallel processing. We use all available cores if not otherwise specified.

\subsection{Results Summary}

We start the presentation of our results by showing measurements averaged over all runs per model, shown in Table 5. This should provide an overview of the results achievable with our techniques and display model characteristics. In total, we performed 6,483 runs across all models in 564 hours of wallclock time. A run means that we started a model with a specific set of parameters.

We report the number mutations introduced per model (Mutants). Further, we present the number of mutants reached (Reach), the number of mutants strongly (SKill) killed, the number of mutants weakly, but not strongy killed (WKill), the number of tests (\# Tests), and average test length (TestL), as well as the wallclock runtimes (Time) in seconds, averaged over the all runs performed per model. Finally, the last column (Runs) shows the overall number of runs we performed for the model.

\subsection{Breadth-First Search}

We started full breadth-first search on all models. Small models work fine and provide useful bounds on the number of mutants that can be reached, as well as how many steps are maximally necessary to do so. For larger models, full breadth-first search did not terminate. Either we reached 
Table 6. Full Breadth-First Search Results

\begin{tabular}{lrrrr}
\hline Model & States & Depth & Reach & Time \\
ChassisDynoDSL & 14 & 3 & 184 & 0.6 \\
PartCountDSL & 222 & 10 & 588 & 1.7 \\
DemoTopology & $\geq 13129$ & $\geq 3$ & $\geq 160$ & $\mathrm{TO}$ \\
AlarmSystem & 76 & 12 & 810 & 78 \\
Debounce & 560 & 9 & 978 & 215 \\
PartCountUML & 12138 & 12 & 2230 & 1719 \\
Defibrillator & $\geq 18580118$ & $\geq 299$ & $\geq 1428$ & $\mathrm{TO}$ \\
Loader & $\geq 1702$ & $\geq 2$ & $\geq 676$ & $\mathrm{MO}$ \\
MMS & $\geq 266112$ & $\geq 4$ & $\geq 1275$ & $\mathrm{MO}$ \\
LBT & $\geq 118413$ & $\geq 2$ & $\geq 365$ & $\mathrm{MO}$ \\
\hline
\end{tabular}

more and more new states and aborted search after 24 hours (TO) or we ran out of memory (MO). These experiments were performed on Machine 2.

We present the relevant measurements for breadth-first searches in Table 6. We report times for runs that finished and TO or MO for unfinished runs. The measurements of unfinished runs represent the results before termination. The column States shows the number of states reached and the column Depth shows the maximal shortest path from the initial state to some state in terms of number of exploration steps. Columns Reach and Time report the number of mutants reached and runtime in seconds, as in the previous Table 5. For unfinished runs, we report the results of the maximal depth fully explored and denote the results with $\geq$ symbols.

We use the results of full breadth-first search to classify our models into small, large, and LBT. Small models are those where BFS finished, i.e., models ChassisDynoDSL, PartCountDSL, AlarmSystem, Debounce, and PartCountUML. Large models are all the others, except LBT. For such models, runs with realistic values for MAXSTEPs terminate within a few hours. LBT is special in its computational demands. We needed to set MAXSTEPS value to 500 and limit the mutants to 500 to get runs that terminate within reasonable time. We did not extensively evaluate heuristics on LBT but demonstrate that our algorithm is able to process this huge model within reasonable time (7 hours per run on Machine 3).

\subsection{Heuristic Evaluation on Small Models}

For small models, we tested the whole cross product of all choices for CreateTask and SelectSuCCESSOR, four different random seeds, three values for MAXSTEPS, as well as nine values for BRANCHLEngth. We chose the bucket select (.) function for all experiments, since it outperformed the other two options in preliminary experiments. For the MAXSTEPS parameter, we chose the number of states $|S|,|S| / 2$, and $|S| / 10$, where $|S|$ was determined with full BFS. For the BranchLength parameter for each MAXSTEPS value, we tested MAXSTEPs, MAXSTEPS/5, and MAXSTEPs/20, resulting in a total of nine parameter combinations. These experiments were performed on Machine 2.

In Table 7, we show the mean results in terms of number of mutants reached achieved by the heuristics with the different parameter values for MAXSTEPS in relation to the results of full BFS. We can see that the heuristics often manage to come close to the BFS results, even for smaller MAXSTEPs values. The last column for ChassisDynoDSL is empty, because the model is too small for sensible experiments with MAXSTEPS $=|\mathbf{S}| / \mathbf{1 0}$. Similarly, the last column in AlarmSystem corresponds to runs with just 7 steps, explaining the relatively poor performance. If we take the maximum, instead of average results, then all table entries but three are $100 \%$ for MAXSTEPS $=|\mathrm{S}| / \mathbf{1 0}$, 
Table 7. Mutants Reached on Small Models in Relation to MAXStEPS, Relative to Full BFS

\begin{tabular}{lrrr}
\hline Model & MAXSTEPS $=|\mathrm{S}|$ & MAXSTEPS $=|\mathrm{S}| / 2$ & MAXSTEPS $=|\mathrm{S}| / \mathbf{1 0}$ \\
ChassisDynoDSL & $99.4 \%$ & $97 \%$ & - \\
PartCountDSL & $86.6 \%$ & $81.6 \%$ & $61.9 \%$ \\
AlarmSystem & $93.0 \%$ & $86.5 \%$ & $47.1 \%$ \\
Debounce & $100.0 \%$ & $100 \%$ & $82.4 \%$ \\
PartCountUML & $98.9 \%$ & $97.3 \%$ & $92.3 \%$ \\
\hline
\end{tabular}

Table 8. Heuristics Performance on Small Models and MAXStEPS $=|\mathrm{S}| / 2$

\begin{tabular}{llllllll}
\hline & \multicolumn{3}{c}{ CREATETASK } & \multicolumn{5}{c}{ SELECTSUCCESSOR } \\
\hline & Mean & \# Best & \# Bad & & Mean & \# Best & \# Bad \\
CGoal & $96.9 \%$ & 1 & 0 & Dist & $93.1 \%$ & 1 & 1 \\
RGoal & $94.9 \%$ & 2 & 1 & RoRoSS & $92.7 \%$ & 2 & 1 \\
P(Rare) & $94.4 \%$ & 2 & 1 & RandSS & $92.6 \%$ & 2 & 1 \\
RandCT & $94.3 \%$ & 1 & 0 & Part & $92.5 \%$ & 3 & 2 \\
P(Uniq) & $94.2 \%$ & 2 & 1 & LocalBFS & $92.4 \%$ & 2 & 3 \\
RoRoCT & $90.6 \%$ & 1 & 3 & & & & \\
Init & $86.3 \%$ & 2 & 3 & & & & \\
\hline
\end{tabular}

where AlarmSystem, PartCountDSL, and PartCountUML have values 79.4\%, 74.7\%, and 99.8\%, respectively. This shows that the heuristics can achieve good results with low effort.

In Table 8, we report the performance of individual heuristics for MAXSTEPS $=|\mathrm{S}| / 2$. We chose MAXSTEPS $=|\mathrm{S}| / 2$, because we want to evaluate how well heuristics perform on a limited budget. The relative results for MAXSTEPS $=|\mathrm{S}| / \mathbf{1 0}$ are similar, but the variability increases. Column Mean shows the average percentage of mutants reached in comparison to the number of mutants reached by BFS. The other two columns are the result of a statistical analysis, iteratively filtering the dataset. We evaluate performance using a one-sided Mann-Whitney U test [39] with a $\mathrm{p}$-value $p=0.05$. The test compares two sets of data and estimates how likely it is that the two sets are equally distributed, in our case with respect to the number of mutants reached. Starting from the full set of runs, we iteratively filter out runs corresponding to significantly under-performing heuristics. In every iteration and for every heuristic that remains in the dataset, we split it into runs using that heuristic and all other runs. We record which heuristic was least likely to be as good as its complement-set and proceed with the respective complement-set. Columns \# Best show for how many models the respective heuristic was among the remaining heuristics when filtering out until all heuristics are equally likely to perform as well as the others. Columns \# Bad show for how many models the respective heuristic was thrown out during filtering by the statistical test.

We can see that the impact of CREATETASK heuristics is larger than the impact of SELECTSUCCESSOR heuristics, as the mean results of the best and worst heuristics are further apart. CGoal seems to be the best choice in the CREATETASK category. RGoal, P(Uniq), P(Rare), and RandCT perform roughly equally well. However, Init and RoRoCT are significantly worse than the other heuristics on the small models. All heuristics are among the best ones in at least one model, showing that all heuristics can be useful on different models. The fact that CGoal performs better than RGoal shows that careful goal selection is important for good search results.

For the SELECTSUCCESSOR category, all the heuristics perform more or less equally well overall. However, Part and LocalBFS are significantly worse than other heuristics on more models. The 

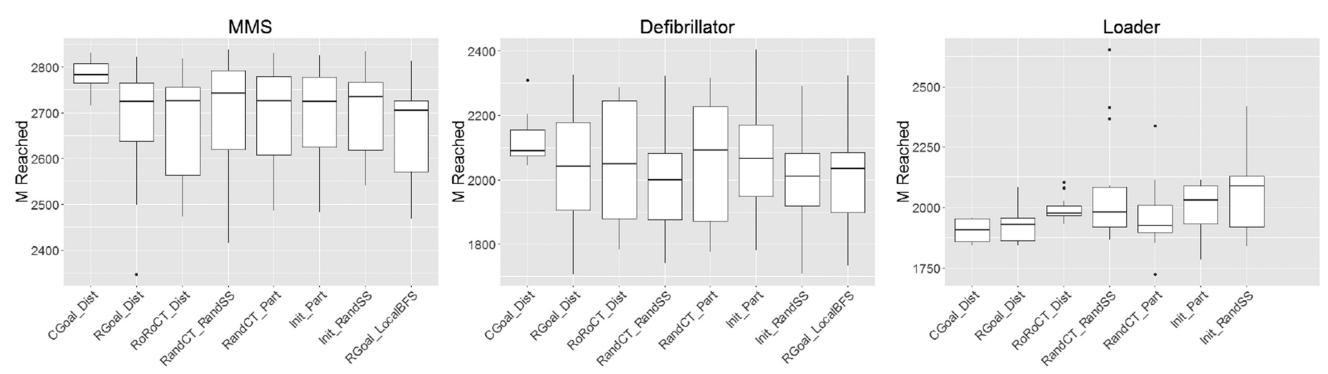

Fig. 2. SelectSuccessor and CreateTask evaluation on large models.

fact that Dist cannot beat random-based strategies more decisively, as would be predicted by the results of RRT in the path planning domain, indicates that a more accurate distance metric is necessary to better reflect mutation reaching capability.

\subsection{Heuristic Evaluation on Large Models}

As mentioned above, testing all combinations of heuristics is infeasible for large models. Therefore, we made a selection of heuristic combinations, based on the data of the small models. To this end, we ranked each combination of CREATETASK and SELECTSUCCESSOR with their mean result and selected combinations that were performing particularly badly (Init $\times$ Part, Init $\times$ RandSS, and RGoal $\times$ LocalBFS), averagely (RandCT $\times$ RandSS, RandCT $\times$ Part, and RoRoCT $\times$ Dist), and well $($ RGoal $\times$ Dist and CGoal $\times$ Dist). We fixed the MAXSTEPS parameter to 100000, 3500, 2000 and 400 for Defibrillator, MMS, Loader, and DemoTopology, respectively. BRANCHLENGTH was set to MAXSteps/5 and MAXSteps/20. These values were determined from the incomplete full BFS runs over these models as a tradeoff between runtime and number of mutants reached. These experiments were performed on Machine 2.

Figure 2 shows the result of large models as a box-plot of the number of mutants reached in relation to the combination of heuristics. Note that the plot scales from minimum number of mutants reached to the maximum number of mutants reached. The DemoTopology model was excluded from this figure, because we found that every heuristic is able to reach all mutants within 400 exploration steps. In that sense, it classifies as a small model. However, as we see in the full BFS results, it has many states, making it a large model.

Like on small models, CGoal performs well on MMS and Defibrillator, not only producing the best mean result, but also showing much less variability than the other heursitics. Interestingly, the heuristic performs worst on Loader in terms of mean result. This underlines again, that multiple heuristics have merit, and there is no perfect heuristic for every model. Init and Part seem to perform better than predicted on small models. For Part this makes sense, since the number of objects increases in larger models, which naturally increases the power of the heuristic. Init performs reasonably on larger models. It seems that when branches are long enough, it can make sense to restart the search at the initial state. For LocalBFS, we had to reduce the depth from 4 to 2 of each local BFS search, in order not to blow up the search space. We had to exclude LocalBFS for Loader altogether, because even a breadth-first search with depth 2 is not feasible within reasonable time on this model.

\subsection{Branching}

We evaluated the influence of the BRANCHLENGTH parameter on search results, by an experiment with different values on two large models, MMS and Defibrillator. We chose these models, because 

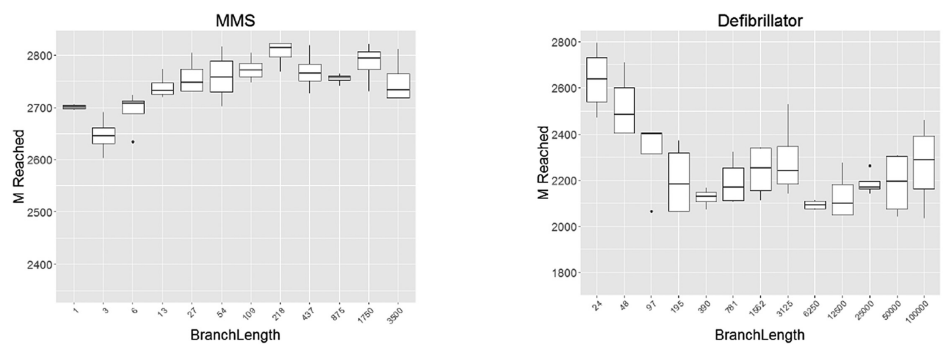

Fig. 3. Mutations reached with multiple parameter values for BRANCHLENGTH.

Table 9. Test Characteristics on Defibrillatorin Relation to MAXSTEPS

\begin{tabular}{rrrrrr}
\hline MAXSTEPS & Graph Trans & Test Trans & Duplicate Trans & Greedy/ShortestP & Time \\
100 & 683 & 334 & 107 & 3.1 & 0.002 \\
200 & 1290 & 467 & 143 & 6.8 & 0.006 \\
1000 & 4342 & 659 & 138 & 11.3 & 0.042 \\
10000 & 21013 & 1581 & 260 & 51.3 & 0.524 \\
20000 & 28772 & 1918 & 271 & 70.5 & 2.420 \\
50000 & 49252 & 2117 & 320 & 109.1 & 17.217 \\
100000 & 63001 & 1904 & 291 & 160.2 & 231.032 \\
\hline
\end{tabular}

they are big enough so there is a lot of variation in the number of mutants reached, but the runs are fast enough so we are able to obtain many data points.

To test the parameter, we set up the following experiment. We set MAXSTEPS to 3500 and 100000 for MMS and Defibrillator respectively. We used RandCT and RandSS, as well as four different random seeds for each value for BRANCHLENGTH. The experiment was performed on Machine 1. Figure 3 shows the result of the experiments. For MMS medium branch lengths work best. For Defibrillator short branch lengths work best. Both results suggest that branching search is indeed justified.

\subsection{Test Case Generation}

We evaluate the test-case generation algorithm and the produced tests with increasing graph size. To this end, we ran an experiment using the Defibrillator model on Machine 1. We iteratively increase MAXSTEPS to create larger and larger IOTS and record test characteristics. The results of this experiment are shown in Table 9. Column Graph Trans shows the size of the final exploration graph in number of transitions. Unsurprisingly, the size of the IOTS increases as we increase the MAXSTEPs parameter. Column Test Trans shows the number of transitions of the final test suite. The number of test transitions does not grow as much as the exploration graph. This can be explained by the fact that after some point, no more mutants can be killed. However, it also shows that doing more exploration beforehand does not harm the final test suite by introducing unnecessary transitions. Column Duplicate Trans shows the number of transitions in the test suite that are taken at least twice. Initially, the ratio between duplicate transitions and test transitions is quite high. This ratio goes down with higher MAXSTEPS values, showing again that exploring more is better. Column Greedy/ShortestP shows how much smaller the test suite created by shortest path-based test-case generation over greedy-based test-case generation is in terms of number of transitions. Shortest path test-case generation is clearly superior to greedy test-case generation. 
While tests created with a shortest path method do not suffer from larger exploration graphs, the greedy method does, as can be seen by the increasing ratio between greedy and shortest path. While for smaller MAXSTEPS the shortest path method is faster, for MAXSTEPS $=100,000$ greedy test-case generation takes only $37 \%$ of the time of the shortest path method. Column Time shows the time in seconds needed for shortest path-based test-case generation, once the final exploration graph is finalized. We can see that test-case generation on large graphs takes a non-negligible time. For example for MAXSTEPS $=100,000$ the search took 13 minutes and the test-case generation took almost 4 minutes. However, for smaller graphs the amount of time spent is low.

\subsection{Parallelization}

We evaluated how much speedup we could achieve by performing exploration steps in parallel. We define speedup as the fraction of average wall-clock time of parallel runs and the average wallclock time of sequential runs. More specifically, we compare total runtimes of executions with 1 thread (sequential) to threads with 20 threads (parallel). We evaluated the speedup on all models but LBT, as running LBT on a single core is infeasible within a reasonable amount of time. For each model, we fix MaxSteps and set BranchLength to MaxSteps/20. Furthermore, we repeat the runs on each model using four different random seeds. The experiments were performed on Machine 1.

Unsurprisingly, the larger models benefit more from parallelization. More precisely, models where one exploration step is computationally expensive benefit more. The model where a single exploration step takes the longest is Loader with $415 \mathrm{~s}$ per step on average. For Loader, we get a speedup of 11.3x. On the other extreme, the average exploration step on PartCountDSL takes only $0.06 \mathrm{~s}$. This model in fact suffers from the synchronization overhead of the parallel implementation and has a speedup of $0.9 x$. For large models, we have an average speedup of 5.98x. Small models do not benefit much from parallelization, having an average speedup of $1.82 \mathrm{x}$.

\section{THREATS TO VALIDITY}

Randomness. Many of our methods are based on random decisions, making them susceptible to random variation. We counter this threat by performing each experiment setup with 4 random seeds and across multiple models. Overall, the validity of the results is based on over 5,000 experiments and thus should not be threatened by randomness. Furthermore, where applicable, we base our conclusions on widely recognised statistical tests.

Bugs. Software contains bugs. Our test-case generation tool is software. It would be naïve to think that our tool is free of bugs. However, our tool is fairly mature and, we have a thorough software quality process, which helps us to mitigate effects of bugs and to detect them early. Furthermore, we performed positive cross checks of produced tests on the models to ensure that we do not produce invalid tests. The tests are used by industrial partners, who report problems if they occur.

Choice of models. All the models used for the experiments are derived from real world use cases and, we did not leave out models available to us. In fact, it would have been easy to construct models of any desired size, such that any desired heuristic performs particularly well. The experiments show that these our models are quite diverse in their computational demands and that different heuristics work well on them.

\section{CONCLUSION}

In this work, we present a heuristic-guided branching search algorithm for discrete and nondeterministic models. We show how strong killing analysis can be combined with the search and how we can generate test cases from search results. We perform an extensive evaluation of the 
presented heuristics and parameters. Our case study models range from very small to very large and were created in an industrial context. Thereby, we demonstrate that our approach scales to realistic problems. We found that multiple heuristics perform well on different models, where some heuristics perform better overall. Furthermore, we demonstrate that the main idea of the search algorithm, i.e., performing search in a branching style, works well. Via branching, we find more mutants and due to parallelization we find them faster.

Finding Best Parameters. Based on our experiments, we describe an approach to finding the best parameters for our search on a new model. First, fix the time budget that is acceptable for test-case generation. Whether seconds, hours or days are acceptable makes a significant difference in what can be expected from the search. Second, perform full BFS on the model and see whether it terminates. If it does, then no other search option has to be tried. Third, check how many steps full BFS has performed until the desired time budget was exhausted and set MAXSTEPS to this value. Fourth, perform a few runs, using the CGoal / Dist heuristic pair with different BRANCHLENGTH parameters to see whether more mutants can be found with small, medium, or long branch parameters. Fifth, try different heuristic pairs. RandCT / RandSS can work well when not much structure of the model can be used. CGoal / Part can work well when the model uses much object orientation.

Future Work. We want to create and leverage constraints expressing reachability of mutants. Such constraints could be used as a distance metric, or to combine heuristic-based search with symbolic approaches. Heuristic-based search can find most mutants cheaply, while symbolic techniques, such as dynamic symbolic execution, can find remaining mutants by calculating solutions to the reachability constraints.

\section{REFERENCES}

[1] Jean-Raymond Abrial. 2010. Modeling in Event-B-System and Software Engineering. Cambridge University Press.

[2] B. Aichernig, H. Brandl, E. Jöbstl, W. Krenn, R. Schlick, and S. Tiran. 2015. MoMuT::UML model-based mutation testing for UML. In Proceedings of the 2015 IEEE 8th International Conference on Software Testing, Verification and Validation (ICST'15).

[3] Bernhard K. Aichernig, Jakob Auer, Elisabeth Jöbstl, Robert Korošec, Willibald Krenn, Rupert Schlick, and Birgit Vera Schmidt. 2014. Model-based mutation testing of an industrial measurement device. In Proceedings of the International Conference on Tests and Proofs. Springer, 1-19.

[4] Bernhard K. Aichernig, Harald Brandl, Elisabeth Jöbstl, Willibald Krenn, Rupert Schlick, and Stefan Tiran. 2015. Killing strategies for model-based mutation testing. Softw. Test., Verif. Reliab. 25, 8 (2015), 716-748.

[5] Bernhard K. Aichernig, Elisabeth Jöbstl, and Stefan Tiran. 2015. Model-based mutation testing via symbolic refinement checking. Sci. Comput. Program. 97 (2015), 383-404. DOI : http://dx.doi.org/10.1016/j.scico.2014.05.004

[6] Baris Akgun and Mike Stilman. 2011. Sampling heuristics for optimal motion planning in high dimensions. In Proceedings of the 2011 IEEE/RSF International Conference on Intelligent Robots and Systems (IROS'11). IEEE.

[7] James H. Andrews, Lionel C. Briand, and Yvan Labiche. 2005. Is mutation an appropriate tool for testing experiments? In Proceedings of the 27th International Conference on Software Engineering (ICSE'05), Gruia-Catalin Roman, William G. Griswold, and Bashar Nuseibeh (Eds.). ACM, 402-411.

[8] John Aycock. 2003. A brief history of just-in-time. ACM Comput. Surv. 35, 2 (2003), 97-113.

[9] Ralph-Johan Back and Reino Kurki-Suonio. 1983. Decentralization of process nets with centralized control. In Proceedings of the 2nd Annual ACM SIGACT-SIGOPS Symposium on Principles of Distributed Computing (PODC'83). ACM.

[10] Marcello M. Bonsangue, Joost N. Kok, and Kaisa Sere. 1998. An approach to object-orientation in action systems. In Proceedings of the Mathematics of Program Construction (MPC'98), Johan Jeuring (Ed.), Vol. 1422. Springer, 68-95. http://link.springer.de/link/service/series/0558/bibs/1422/14220068.htm.

[11] Michael S. Branicky, Michael M. Curtiss, Joshua A. Levine, and Stuart B. Morgan. 2003. RRTs for nonlinear, discrete, and hybrid planning and control. In Proceedings of the 42nd IEEE Conference on Decision and Control, Vol. 1. IEEE, 657-663.

[12] Manfred Broy, Bengt Jonsson, Joost-Pieter Katoen, Martin Leucker, and Alexander Pretschner. 2005. Model-based Testing of Reactive Systems: Advanced Lectures. Vol. 3472. Springer.

[13] Timothy A. Budd, Richard J. Lipton, Richard A. DeMillo, and Frederick G. Sayward. 1979. Mutation Analysis. Technical Report. DTIC Document. 
[14] Tsong Yueh Chen, Fei-Ching Kuo, Robert G. Merkel, and T. H. Tse. 2010. Adaptive random testing: The art of test-case diversity. F. Syst. Softw. 83, 1 (2010), 60-66.

[15] Tsun S. Chow. 1978. Testing software design modeled by finite-state machines. IEEE Trans. Softw. Eng. 4, 3 (1978).

[16] Ilinca Ciupa, Andreas Leitner, Manuel Oriol, and Bertrand Meyer. 2008. ARTOO: Adaptive random testing for objectoriented software. In Proceedings of the 30th International Conference on Software Engineering. ACM, 71-80.

[17] Arilo C. Dias Neto, Rajesh Subramanyan, Marlon Vieira, and Guilherme H. Travassos. 2007. A survey on modelbased testing approaches: A systematic review. In Proceedings of the Workshop on Empirical Assessment of Software Engineering Languages and Technologies. 31-36.

[18] Edsger W. Dijkstra. 1975. Guarded commands, nondeterminacy and formal derivation of programs. Commun. ACM 18, 8 (1975), 453-457.

[19] Tommaso Dreossi, Thao Dang, Alexandre Donzé, James Kapinski, Xiaoqing Jin, and Jyotirmoy V. Deshmukh. 2015. Efficient guiding strategies for testing of temporal properties of hybrid systems. In Proceedings of the NASA Formal Methods Symposium. Springer, 127-142.

[20] Andreas Fellner, Willibald Krenn, Rupert Schlick, Thorsten Tarrach, and Georg Weissenbacher. 2017. Model-based, mutation-driven test case generation via heuristic-guided branching search. In Proceedings of the 15th ACM-IEEE International Conference on Formal Methods and Models for System Design (MEMOCODE'17). ACM.

[21] Dave Ferguson, Nidhi Kalra, and Anthony Stentz. 2006. Replanning with RRTs. In Proceedings of the 2006 IEEE International Conference on Robotics and Automation (ICRA'06). IEEE.

[22] Gordon Fraser and Andrea Arcuri. 2011. Evolutionary generation of whole test suites. In Proceedings of the 11th International Conference on Quality Software (QSIC'11), Manuel Núñez, Robert M. Hierons, and Mercedes G. Merayo (Eds.). IEEE Computer Society, 31-40. DOI : http://dx.doi.org/10.1109/QSIC.2011.19

[23] Gordon Fraser and Andreas Zeller. 2012. Mutation-driven generation of unit tests and oracles. IEEE Trans. Softw. Eng. 38, 2 (2012), 278-292.

[24] Matthew J. Gallagher and V. Lakshmi Narasimhan. 1997. Adtest: A test data generation suite for ada software systems. IEEE Trans. Softw. Eng. 23, 8 (1997), 473-484.

[25] Patrice Godefroid, Nils Klarlund, and Koushik Sen. 2005. DART: Directed automated random testing. In ACM Sigplan Notices, Vol. 40. ACM, 213-223.

[26] Christoph Hilken and Jan Peleska. 2015. Model-based testing against complex SysML models. In Proceedings of the Formal Modeling and Verification of Cyber-Physical Systems, and the 1st International Summer School on Methods and Tools for the Design of Digital Systems, Rolf Drechsler and Ulrich Kühne (Eds.). Springer, 284-286.

[27] William E. Howden. 1982. Weak mutation testing and completeness of test sets. IEEE Trans. Softw. Eng. 8, 4 (1982).

[28] Leonard Jaillet, Juan Cortés, and Thierry Siméon. 2008. Transition-based RRT for path planning in continuous cost spaces. In Proceedings of the 2008 IEEE/RSf International Conference on Intelligent Robots and Systems. IEEE.

[29] Claude Jard and Thierry Jéron. 2005. TGV: Theory, principles and algorithms. Int. f. Softw. Tools Technol. Transf. 7, 4 (2005), 297-315.

[30] Yue Jia and Mark Harman. 2011. An analysis and survey of the development of mutation testing. IEEE . Softw. Eng. 37, 5 (2011), 649-678. http://ieeexplore.ieee.org/xpls/abs_all.jsp? arnumber=5487526.

[31] René Just, Darioush Jalali, Laura Inozemtseva, Michael D. Ernst, Reid Holmes, and Gordon Fraser. 2014. Are mutants a valid substitute for real faults in software testing? In Proceedings of the 22nd ACM SIGSOFT International Symposium on Foundations of Software Engineering (FSE-22). ACM.

[32] Bogdan Korel. 1990. Automated software test data generation. IEEE Trans. Softw. Eng. 16, 8 (1990).

[33] Willibald Krenn, Rupert Schlick, and Bernhard K. Aichernig. 2009. Mapping UML to labeled transition systems for test-case generation: A translation via object-oriented action systems. In Proceedings of the 8th International Conference on Formal Methods for Components and Objects (FMCO'09). Springer-Verlag, Berlin, 186-207.

[34] James J. Kuffner and Steven M. LaValle. 2000. RRT-connect: An efficient approach to single-query path planning. In Proceedings of the IEEE International Conference on Robotics and Automation (ICRA'00), Vol. 2. IEEE, 995-1001.

[35] Chris Lattner and Vikram S. Adve. 2004. LLVM: A compilation framework for lifelong program analysis \& transformation. In Proceedings of the 2nd IEEE / ACM International Symposium on Code Generation and Optimization (CGO'04)

[36] Steven M. LaValle. 1998. Rapidly-Exploring Random Trees: A New Tool for Path Planning. Technical Report.

[37] Lei Ma, Cyrille Artho, Cheng Zhang, Hiroyuki Sato, Johannes Gmeiner, and Rudolf Ramler. 2015. GRT: Programanalysis-guided random testing (T). In Proceedings of the 30th IEEE/ACM International Conference on Automated Software Engineering (ASE'15). 212-223.

[38] Jan Malburg and Gordon Fraser. 2011. Combining search-based and constraint-based testing. In Proceedings of the 26th IEEE/ACM International Conference on Automated Software Engineering (ASE'11) (ASE), Perry Alexander, Corina S. Pasareanu, and John G. Hosking (Eds.). IEEE Computer Society, 436-439.

[39] Henry B. Mann and Donald R. Whitney. 1947. On a test of whether one of two random variables is stochastically larger than the other. Ann. Math. Stat. 18, 1 (1947), 50-60. http://www.jstor.org/stable/2236101. 
[40] Phil McMinn. 2004. Search-based software test data generation: A survey. Softw. Test. Verif. Reliabil. 14, 2 (2004), 105-156.

[41] Carlos Pacheco and Michael D. Ernst. 2007. Randoop: Feedback-directed random testing for Java. In Proceedings of the Companion to the 22nd Annual ACM SIGPLAN Conference on Object-Oriented Programming, Systems, Languages, and Applications (OOPSLA'07). 815-816.

[42] Carlos Pacheco, Shuvendu K. Lahiri, Michael D. Ernst, and Thomas Ball. 2007. Feedback-directed random test generation. In Proceedings of the 29th International Conference on Software Engineering (ICSE'07). IEEE Computer Society, $75-84$.

[43] Alexander Pretschner, Wolfgang Prenninger, Stefan Wagner, Christian Kühnel, Martin Baumgartner, Bernd Sostawa, Rüdiger Zölch, and Thomas Stauner. 2005. One evaluation of model-based testing and its automation. In Proceedings of the 27th International Conference on Software Engineering (ICSE'05). ACM, 392-401.

[44] Klaus Reichl, Tomas Fischer, and Peter Tummeltshammer. 2016. Using formal methods for verification and validation in railway. In Proceedings of the 10th International Conference onTests and Proofs (TAP'16), Bernhard K. Aichernig and Carlo A. Furia (Eds.), Lecture Notes in Computer Science, Vol. 9762. Springer.

[45] Manoranjan Satpathy, Anand Yeolekar, and S. Ramesh. 2008. Randomized directed testing (REDIRECT) for Simulink/Stateflow models. In Proceedings of the 8th ACM International Conference on Embedded Software. ACM, $217-226$.

[46] Muhammad Shafique and Yvan Labiche. 2010. A Systematic Review of Model Based Testing Tool Support. Carleton University, Canada, Tech. Rep. Technical Report SCE-10-04 (2010), 01-21.

[47] Muhammad Shafique and Yvan Labiche. 2015. A systematic review of state-based test tools. Softw. Tools Technol. Transf. 17, 1 (2015), 59-76.

[48] Nigel Tracey, John Clark, Keith Mander, and John McDermid. 1998. An automated framework for structural test-data generation. In Proceedings of the 13th IEEE International Conference on Automated Software Engineering (ASE'98). IEEE.

[49] Gerrit Jan Tretmans. 1992. A Formal Approach to Conformance Testing. Ph.D. Dissertation. University of Twente, Enschede, Netherlands.

[50] Jan Tretmans. 1996. Test generation with inputs, outputs and repetitive quiescence. Softw. Concept. Tools 17, 3 (1996), $103-120$.

[51] Mark Utting, Alexander Pretschner, and Bruno Legeard. 2012. A taxonomy of model-based testing approaches. Softw. Test. Verif. Reliabil. 22, 5 (2012), 297-312.

[52] Mattia Vivanti, Andre Mis, Alessandra Gorla, and Gordon Fraser. 2013. Search-based data-flow test generation. In Proceedings of the 2013 IEEE 24th International Symposium on Software Reliability Engineering (ISSRE'13). IEEE, 370379.

[53] Joachim Wegener, Kerstin Buhr, and Hartmut Pohlheim. 2002. Automatic test data generation for structural testing of embedded software systems by evolutionary testing. In Proceedings of the Annual Conference on Genetic and Evolutionary Computation (GECCO'02), Vol. 2. 1233-1240.

[54] Yuan Zhan and John A. Clark. 2005. Search-based mutation testing for simulink models. In Proceedings of the 7th Annual Conference on Genetic and Evolutionary Computation (GECCO'05). ACM, New York, NY, 1061-1068.

Received January 2018; revised July 2018; accepted October 2018 